Research Article

\title{
Fixed-Time Synchronization of Delayed Memristive Neural Networks with Discontinuous Activations
}

\author{
Hao Pu $\mathbb{D}^{1,2}$ and Fengjun $\mathrm{Li} \mathbb{D}^{1}$ \\ ${ }^{1}$ School of Mathematics and Statistics, Ningxia University, Yinchuan 750021, Ningxia, China \\ ${ }^{2}$ School of Mathematics, Zunyi Normal College, Zunyi 563000, Guizhou, China \\ Correspondence should be addressed to Fengjun Li; fjli@nxu.edu.cn
}

Received 18 May 2021; Revised 5 June 2021; Accepted 15 June 2021; Published 13 July 2021

Academic Editor: Niansheng Tang

Copyright @ $2021 \mathrm{Hao} \mathrm{Pu}$ and Fengjun Li. This is an open access article distributed under the Creative Commons Attribution License, which permits unrestricted use, distribution, and reproduction in any medium, provided the original work is properly cited.

\begin{abstract}
In this paper, the fixed-time synchronization problem for a class of memristive neural networks with discontinuous neuron activation functions and mixed time-varying delays is investigated. With the help of the fixed-time stability theory, under the framework of Filippov solution and differential inclusion theory, several new and useful sufficient criteria for fixed-time synchronization are obtained by designing two types of energy-saving and simple controllers for the considered systems. Compared with the traditional fixed-time synchronization controller, the controllers used in this paper only have one power exponent term, which is a function of the system state error rather than a constant. Moreover, some previous relevant works are especially improved. Finally, two numerical examples are given to show the correctness and the effectiveness of the obtained theoretical results.
\end{abstract}

\section{Introduction}

The memristor, first introduced by Chua in 1971 [1], is a kind of nonlinear resistor with memory function for the amount of charge, which shows the relationship between the charge and magnetic flux. The first nanomemristor device was created in 2008 by HP engineers [2]. Since then, it has been applied in artificial neural networks to simulate artificial synapse for biological signal processing in that it has a better memory than resistors and can change its performance in response to the voltage and current like a biological synapse [3-5]. As everyone knows, the memristive neural network is a memristor-based neural network. Nowadays, the memristor-based neural network, applied in many fields such as cryptography, combinatorial optimization, associative memory, nonvolatile memory, signal processing, information storage, secure communication, and simulation of biological brains, has become a topic of wide concern and further study [6-10].

In the past few decades, researchers have carried out indepth studies on the dynamics of different neural networks, especially the synchronization behavior that has received considerable attention [11-14]. Synchronization, which means that the systems achieve identical state behaviors over time, is common in nature and is considered to play an important role in many fields of climatology, intelligent control, pattern recognition, chemical reaction, and so forth. It is well known that there are many different types of synchronization concepts in applications, including bipartite synchronization, exponential synchronization, weighted sum synchronization, projection synchronization, preassigned-time synchronization, finite-time synchronization, and fixed-time synchronization [15-22].

Memristive neural network synchronization is segregated into infinite-time synchronization and finite-time synchronization based on the convergence speed. According to previous works $[16-18,20]$, synchronization can be realized when time $t$ approaches infinity. However, it is necessary to achieve synchronization in a finite time in many fields like engineering and science to save cost and improve efficiency $[15,23]$. For instance, the finite-time synchronization of complex-valued memristive-based neural networks 
was discussed by designing a decentralized finite-time synchronization controller [15]. From the above studies $[15,23]$, one can find that finite time $T$ was a function of and changed along with the initial value of the system, which cannot be determined in advance and might bring trouble to the solvency of practical problems. Fortunately, in 2012, the fixed-time stability theory was proposed for the first time by Polyakov, making the settling time $T$ independent of the initial value of the system [24]. Since it has been widely applied in scientific research and engineering management, researchers became more and more interested in it and put forward better theories of fixed-time stability, which reduced the conservatism and reflected the actual stable time more accurately. To give an example, a new theorem of fixed-time stability was established and the settling time was precisely calculated in [22]. As the fixed-time synchronization of the system has a higher convergence speed and better performance on interference suppression, the control for synchronization of memristive neural networks has attracted much attention from researchers [25-29]. For example, in [27], the authors discussed the fixed-time synchronization of memristive fuzzy BAM cellular neural networks with timevarying delays based on the Lyapunov stability theory. The fixed-time synchronization of coupled memristor-based neural networks with time-varying delays was discussed in [29] via inequality techniques, the nonsmooth analysis theory, novel state-feedback controllers, and an adaptive controller.

It is not hard to notice that, from the previous literature review, many published works on neural network synchronization focused on the hypothesis that the activation function should be continuous, bounded, or even global Lipschitz. However, most neural networks employed in science and engineering such as dry friction, power circuits, and switching off electronics are using discontinuous neuron activation functions, which are ideal for solving linear programming problems and constrained optimization problems $[30,31]$. Therefore, in recent years, neural networks with discontinuous neuron activation function have attracted considerable attention and many good results have been obtained [32-36]. For example, under the framework of the Filippov solution, the new exponential synchronization criteria for time-varying delayed neural networks with discontinuous activations were investigated by designing a discontinuous state-feedback controller in [33]. The authors investigated the fixed-time synchronization for coupled delayed neural networks with discontinuous or continuous activations in [35].

It is well known that discrete delays are inevitably encountered in neural networks during the signal transmission among the neurons due to the finite switching speed of neurons and amplifiers. At the same time, the neural network is spatial. There are many parallel pathways with different axon sizes and lengths, and the distribution delay should also be considered. Besides, the discrete-time delays and distributed delays often affect the stability of the neural network system and may lead to some complex dynamic behaviors, such as instability, chaos, and oscillation. Thus, the synchronization of memristive neural networks with mixed time-varying delays has been widely concerned [37-39]. The global synchronization of fuzzy memristive neural networks with discrete and distributed delays was investigated in [37] based on the nonsmooth analysis and Lyapunov stability theory. The synchronization of memristive neural networks with mixed time delays was discussed under a quantized intermittent control in [39] with weighted double-integral inequalities and novel Lyapunov-Krasovskii functionals.

As far as we know, there have been only a few studies on the memristive neural network with discontinuous activation functions and mixed time-varying delays [40-43]. The synchronization with a general decay rate for memristorbased BAM neural networks with distributed delays and discontinuous activation functions was discussed in [40] with two different types of nonlinear controllers based on the extended Filippov framework and the theory of differential inclusion. In [41], the authors studied the general decay synchronization of memristor-based Cohen-Grossberg neural networks with mixed time delays and discontinuous activations by applying the Lyapunov-Krasovskii functionals, the concept of Filippov solution, and the theory of differential inclusion. The adaptive synchronization of memristor-based neural networks with discontinuous activations was investigated in [42] based on the extended Filippov framework and the theory of differential inclusion. However, the authors only studied the infinite-time synchronization instead of the fixed-time synchronization which has a higher convergence speed. Unfortunately, the life span of human beings and the service life of the equipment are limited, and it is a common pursuit of people to save costs and improve efficiency. Therefore, the synchronization of memristive neural networks with discontinuous neuron activation functions and mixed time-varying delays needs to be achieved in a fixed time. Yet this issue has not been studied by many researchers [44].

Inspired by the above discussions, the fixed-time synchronization problem for a class of memristive neural networks with discontinuous neuron activation functions and mixed time-varying delays will be discussed. Firstly, the discontinuous activation function and mixed time-varying delays are considered in the model used for this study. Secondly, two energy-saving and simple controllers are designed with only one power exponent term serving as a system state error rather than a constant and the control parameters are closely related to the distributed delay and the state switching jump. Furthermore, several new and useful sufficient conditions of fixed-time synchronization are provided under the fixed-time stability theory, the extended Filippov framework, and the theory of differential inclusion. The settling times of fixed-time synchronization are also estimated and become closer to the actual synchronization time, making improvements on previous works. Finally, two examples of numerical simulations are given to demonstrate the validity of the obtained results.

The rest of this paper is organized into five sections. Model descriptions and preliminaries are given in Section 2 and some sufficient conditions for fixed-time synchronization are provided in Section 3. In Section 4, two numerical 
simulations are given to demonstrate the validity of the obtained theoretical results. Finally, the conclusions are drawn in Section 5.

\section{Problem Formulation and Preliminaries}

In this paper, a class of memristive neural networks are considered with discontinuous activation functions and mixed time-varying delays described by the following equation:

$$
\begin{aligned}
\dot{x}_{i}(t)= & -a_{i}\left(x_{i}(t)\right) x_{i}(t)+\sum_{j=1}^{n} b_{i j}\left(x_{i}(t)\right) f_{j}\left(x_{j}(t)\right)+\sum_{j=1}^{n} c_{i j}\left(x_{i}(t)\right) \\
& \times g_{j}\left(x_{j}\left(t-\tau_{j}(t)\right)\right)+\sum_{j=1}^{n} d_{i j}\left(x_{i}(t)\right) \int_{t-\delta_{j}(t)}^{t} f_{j}\left(x_{j}(s)\right) \mathrm{d} s+I_{i},
\end{aligned}
$$

where $i \in I=\{1,2, \ldots, n\}$ and $n \geq 2$ denotes the number of neurons in the neural networks; $x_{i}(t)$ denotes the state variable of the $i$ th neuron at time $t ; f_{j}(\cdot)$ and $g_{j}(\cdot)$ are the activation functions; $\tau_{j}(t)$ and $\delta_{j}(t)$ are the discrete timevarying delay and the distributed time delay, respectively, and, satisfying $\tau_{j}(t) \leq \tau_{j}, 0 \leq \delta_{j}(t) \leq \delta_{j} ; I_{i}$ is the external bias on the $i$ th unit; $a_{i}(\cdot), b_{i j}(\cdot), c_{i j}(\cdot)$, and $d_{i j}(\cdot)$ represent the memristive connection weights, $i, j \in I$.

According to the property of memristor and currentvoltage characteristics, the memristive connection weights can be described as the following mathematical model:

$$
\begin{aligned}
a_{i}\left(x_{i}(t)\right) & = \begin{cases}a_{i}^{*}, & \left|x_{i}(t)\right| \leq T, \\
a_{i}^{* *}, & \left|x_{i}(t)\right|>T,\end{cases} \\
b_{i j}\left(x_{i}(t)\right) & = \begin{cases}b_{i j}^{*}, & \left|x_{i}(t)\right| \leq T_{i}, \\
b_{i j}^{* *}, & \left|x_{i}(t)\right|>T_{i},\end{cases} \\
c_{i j}\left(x_{i}(t)\right) & = \begin{cases}c_{i j}^{*}, & \left|x_{i}(t)\right| \leq T_{i}, \\
c_{i j}^{* *}, & \left|x_{i}(t)\right|>T,\end{cases} \\
d_{i j}\left(x_{i}(t)\right) & = \begin{cases}d_{i j}^{*}, & \left|x_{i}(t)\right| \leq T_{i}, \\
d_{i j}^{* *}, & \left|x_{i}(t)\right|>T_{i},\end{cases}
\end{aligned}
$$

for $i, j \in I$, where $a_{i}^{*}, a_{i}^{* *}, b_{i j}^{*}, b_{i j}^{* *}, c_{i j}^{*}, c_{i j}^{* *}, d_{i j}^{*}, d_{i j}^{* *}$ are known constants, and $T_{i}>0$ are switching jumps. Interested readers can further understand the structure of memristive neural networks by consulting $[2,3,13,17]$.

The initial values of system (1) are given by $x_{i}(s)=\phi_{i}(s), s \in[-r, 0], i \in I$, where $r=\max _{j \in I}\left\{\tau_{j}, \delta_{j}\right\}$.

Throughout this paper, we denote $\underline{a}_{i}=\min \left\{a_{i}^{*}, a_{i}^{* *}\right\}, \bar{a}_{i}$ $=\max \left\{a_{i}^{*}, a_{i}^{* *}\right\}, \widehat{b}_{i j}=\min \left\{b_{i j}^{*}, b_{i j}^{* *}\right\}, \breve{b}_{i j}=\max \left\{b_{i j}^{*}, b_{i j}^{* *}\right\}$, $\bar{b}_{i j}=\max \left\{\left|b_{i j}^{*}\right|,\left|b_{i j}^{* *}\right|\right\}, \quad \widehat{c}_{i j}=\min \left\{c_{i j}^{*}, c_{i j}^{* *}\right\}, \check{c}_{i j}=\max \left\{c_{i j}^{*}\right.$, $\left.c_{i j}^{* *}\right\}, \bar{c}_{i j}=\max \left\{\left|c_{i j}^{*}\right|,\left|c_{i j}^{* *}\right|\right\}, \widehat{d}_{i j}=\min \left\{d_{i j}^{*}, d_{i j}^{* *}\right\}, \check{d}_{i j}=\max$ $\left\{d_{i j}^{*}, d_{i j}^{* *}\right\}, \bar{d}_{i j}=\max \left\{\left|d_{i j}^{*}\right|,\left|d_{i j}^{* *}\right|\right\}, i, j \in I$.

Then it is not difficult to obtain that

$$
\begin{gathered}
K\left[a_{i}\left(x_{i}(t)\right)\right]= \begin{cases}a_{i}^{*}, & \left|x_{i}(t)\right| \leq T_{i}, \\
{\left[\underline{a}_{i}, \bar{a}_{i}\right],} & \left|x_{i}(t)\right|=T, \\
a_{i}^{* *}, & \left|x_{i}(t)\right|>T,\end{cases} \\
K\left[b_{i j}\left(x_{i}(t)\right)\right]= \begin{cases}b_{i j}^{*}, & \left|x_{i}(t)\right| \leq T_{i}, \\
{\left[\widehat{b}_{i j}, \check{b}_{i j}\right],} & \left|x_{i}(t)\right|=T_{i}, \\
b_{i j}^{* *}, & \left|x_{i}(t)\right|>T_{i},\end{cases} \\
K\left[c_{i j}\left(x_{i}(t)\right)\right]= \begin{cases}c_{i j}^{*}, & \left|x_{i}(t)\right| \leq T_{i}, \\
{\left[\widehat{c}_{i j}, \check{c}_{i j}\right],} & \left|x_{i}(t)\right|=T_{i}, \\
c_{i j}^{* *}, & \left|x_{i}(t)\right|>T_{i},\end{cases} \\
K\left[d_{i j}\left(x_{i}(t)\right)\right]= \begin{cases}d_{i j}^{*}, & \left|x_{i}(t)\right| \leq T_{i}, \\
{\left[\widehat{d}_{i j}, \check{d}_{i j}\right],} & \left|x_{i}(t)\right| \leq T_{i}, \\
d_{i j}^{* *}, & \left|x_{i}(t)\right|>T_{i} .\end{cases}
\end{gathered}
$$

To obtain our main results in the next section, for the memristive neural network system (1), it is necessary to introduce the following assumptions:

$\left(H_{1}\right)$ For each $j \in I$, the neuron activation functions $f_{j}(\cdot)$ and $g_{j}(\cdot)$ are continuous on $R$ except a denumerable set of isolate discontinuous points $\left\{\omega_{k}^{j}\right\}$, where there exist finite right and left limits $f_{j}^{+}\left(\omega_{k}^{j}\right), f_{j}^{-}\left(\omega_{k}^{j}\right)$, $g_{j}^{+}\left(\omega_{k}^{j}\right)$, and $g_{j}^{-}\left(\omega_{k}^{j}\right)$.

$\left(H_{2}\right)^{[41]}$ For each $j \in I$, there exist positive constants $L_{j}, N_{j}, Z_{j}$, and $H_{j}$ such that the neuron activation functions $f_{j}(\cdot)$ and $g_{j}(\cdot)$ satisfy

$$
\begin{array}{r}
\sup _{\left.\eta_{i} \in K\left[f_{j}\left(v_{j}\right)\right], \gamma_{i} \in K\left[f_{j}\left(u_{j}\right)\right]\right]_{\infty}}\left|\eta_{j}-\gamma_{j}\right| \leq L_{j}\left|v_{j}-u_{j}\right|+N_{j}, u_{j}, v_{j} \in R, \\
\sup _{\bar{\eta}_{i} \in K\left[g_{j}\left(v_{j}\right)\right], \bar{\gamma}_{i} \in K\left[g_{j}\left(u_{j}\right)\right]}\left|\bar{\eta}_{j}-\bar{\gamma}_{j}\right| \leq Z_{j}\left|v_{j}-u_{j}\right|+H_{j}, u_{j}, v_{j} \in R,
\end{array}
$$


where

$$
\begin{aligned}
K\left[f_{j}(\chi)\right] & =\left[\min \left\{f_{j}^{-}(\chi), f_{j}^{+}(\chi)\right\}, \max \left\{f_{j}^{-}(\chi), f_{j}^{+}(\chi)\right\}\right], \\
K\left[g_{j}(\chi)\right] & =\left[\min \left\{g_{j}^{-}(\chi), g_{j}^{+}(\chi)\right\}, \max \left\{g_{j}^{-}(\chi), g_{j}^{+}(\chi)\right\}\right],
\end{aligned}
$$

for $\chi \in R$.

$\left(H_{3}\right) \forall u \in R$ and $j \in I$, the activation functions $f_{j}$ and $g_{j}$ are bounded. That is, there exist positive constants $S_{j}$ and $M_{j}$ such that

$$
\begin{aligned}
& \left|f_{j}(u)\right| \leq S_{j}, \\
& \left|g_{j}(u)\right| \leq M_{j} .
\end{aligned}
$$

$\left(H_{4}\right) \forall u, v \in R$, there exist positive constants $L_{j}$ and $Z_{j}$ such that the continuous neuron activation functions $f_{j}(\cdot)$ and $g_{j}(\cdot)$ satisfy

$$
\begin{aligned}
& \left|f_{j}(v)-f_{j}(u)\right| \leq L_{j}|v-u|, \\
& \left|g_{j}(v)-g_{j}(u)\right| \leq Z_{j}|v-u|, j \in I .
\end{aligned}
$$

Considering memristive neural network (1) as the drive system, the corresponding controlled response system is expressed by

$$
\begin{aligned}
\dot{y}_{i}(t)= & -a_{i}\left(y_{i}(t)\right) y_{i}(t)+\sum_{j=1}^{n} b_{i j}\left(y_{i}(t)\right) f_{j}\left(y_{j}(t)\right)+\sum_{j=1}^{n} c_{i j}\left(y_{i}(t)\right) \\
& \times g_{j}\left(y_{j}\left(t-\tau_{j}(t)\right)\right)+\sum_{j=1}^{n} d_{i j}\left(y_{i}(t)\right) \int_{t-\delta_{j}(t)}^{t} f_{j}\left(y_{j}(s)\right) \mathrm{d} s+I_{i}+K_{i}(t),
\end{aligned}
$$

where $K_{i}(t)$ denotes the nonlinear state-feedback controller which will be designed later. The memristive connection weights of the response system (8) are defined the same as those of the drive system (1). The initial conditions of the response system $(8)$ are $y_{i}(s)=\varphi_{i}(s), s \in[-r, 0], i \in I$.

Due to the memristive connection weights $a_{i}(\cdot), b_{i j}(\cdot)$, $c_{i j}(\cdot)$, and $d_{i j}(\cdot)$ and the fact that the activation functions $f_{j}(\cdot)$ and $g_{i}(\cdot)$ of the drive-response systems (1) and (8) are discontinuous, the classic solution is not suitable for the drive-response systems (1) and (8). The solutions of the drive-response systems (1) and (8) in the conventional sense do not exist. As far as we know, the Filippov solution is an effective tool to solve the differential equations with the discontinuous right-hand side. Therefore, the following definition of the Filippov solution needs to be introduced.

Definition 1 (see [33]). Consider a system with discontinuous right side in the form of

$$
\begin{aligned}
& \dot{\zeta}(t)=F(\zeta(t)), \\
& \zeta(0)=\zeta_{0}
\end{aligned}
$$

where $\zeta \in R^{m}, F(\zeta): R^{m} \longrightarrow R^{m}$ is locally bounded and Lebesgue measurable. The function $\zeta(t)$ is said to be the solution in Filippov sense defined in the interval $[0, T)$, if $\zeta(t)$ is absolutely continuous and the following differential inclusion holds:

$$
\dot{\zeta}(t) \in K[F](\zeta(t)), \quad \text { a.e. } t \in[0, T),
$$

where the set-valued map $K[F]: R^{m} \longrightarrow R^{m}$ is defined as

$$
K[F](\zeta(t)) \triangleq \cap_{\varrho>0} \cap_{\mu(N)=0} \overline{c o}\{F(B(\zeta(t), \varrho) \backslash N)\},
$$

where $\overline{\mathrm{co}}$ stands for the closed convex closure, $\mu$ is the Lebesgue measure, and $B(\zeta(t), \varrho)$ denotes the open ball centered at $\zeta(t)$ with radius $\varrho$.

From Definition 1, differential inclusion theory, and the drive-response systems (1) and (8), we can derive that

$$
\begin{aligned}
\dot{x}_{i}(t) \epsilon & -K\left[a_{i}\left(x_{i}(t)\right)\right] x_{i}(t)+\sum_{j=1}^{n} K\left[b_{i j}\left(x_{i}(t)\right)\right] K\left[f_{j}\left(x_{j}(t)\right)\right]+\sum_{j=1}^{n} K\left[c_{i j}\left(x_{i}(t)\right)\right] \\
& \times K\left[g_{j}\left(x_{j}\left(t-\tau_{j}(t)\right)\right)\right]+\sum_{j=1}^{n} K\left[d_{i j}\left(x_{i}(t)\right)\right] \int_{t-\delta_{j}(t)}^{t} K\left[f_{j}\left(x_{j}(s)\right)\right] \mathrm{d} s+I_{i}, \\
\dot{y}_{i}(t) \epsilon & -K\left[a_{i}\left(y_{i}(t)\right)\right] y_{i}(t)+\sum_{j=1}^{n} K\left[b_{i j}\left(y_{i}(t)\right)\right] K\left[f_{j}\left(y_{j}(t)\right)\right]+\sum_{j=1}^{n} K\left[c_{i j}\left(y_{i}(t)\right)\right] \\
& \times K\left[g_{j}\left(y_{j}\left(t-\tau_{j}(t)\right)\right)\right]+\sum_{j=1}^{n} K\left[d_{i j}\left(y_{i}(t)\right)\right] \int_{t-\delta_{j}(t)}^{t} K\left[f_{j}\left(y_{j}(s)\right)\right] \mathrm{d} s+I_{i}+K_{i}(t) .
\end{aligned}
$$


Based on the measurable selection theorem [45], if $x_{i}(t)$ and $y_{i}(t)$ are the solutions of the drive-response systems (1) and (8), respectively, then there exist $\tilde{a}_{i}(t) \in K\left[a_{i}\left(x_{i}(t)\right)\right]$, $\widetilde{b}_{i j}(t) \in K\left[b_{i j}\left(x_{i}(t)\right)\right], \widetilde{c}_{i j} \quad(t) \in \quad K\left[c_{i j}\left(x_{i}(t)\right)\right], \widetilde{d}_{i j}$ $(t) \in K\left[d_{i j}\left(x_{i}(t)\right)\right], \quad \eta_{j}(t) \in K\left[f_{j}\left(x_{j} \quad(t)\right)\right], \bar{\eta}_{j}\left(t-\tau_{j}(t)\right)$ $\in K\left[g_{j}\left(x_{j} \quad\left(t-\tau_{j}(t)\right)\right)\right], \quad a_{i}(t) \quad \in K\left[a_{i}\left(y_{i} \quad(t)\right)\right], b_{i j}$ $(t) \in K\left[b_{i j}\left(y_{i}(t)\right)\right], c_{i j}(t) \in K \quad\left[c_{i j}\left(y_{i} \quad(t)\right)\right],{ }^{,} \quad d_{i j}(t)$ $\in K\left[d_{i j}\left(y_{i}(t)\right)\right], \quad$ and $\quad \gamma_{j}(t) \in K\left[f_{j} \quad\left(y_{j}(t)\right)\right], \bar{\gamma}_{j}(t$ $\left.-\tau_{j}(t)\right) \in K\left[g_{j}\left(y_{j}\left(t-\tau_{j}(t)\right)\right)\right]$, satisfying

$$
\begin{aligned}
\dot{x}_{i}(t)= & -\widetilde{a}_{i}(t) x_{i}(t)+\sum_{j=1}^{n} \widetilde{b}_{i j}(t) \eta_{j}(t)+\sum_{j=1}^{n} \widetilde{c}_{i j}(t) \bar{\eta}_{j}\left(t-\tau_{j}(t)\right) \\
& +\sum_{j=1}^{n} \widetilde{d}_{i j}(t) \int_{t-\delta_{j}(t)}^{t} \eta_{j}(s) \mathrm{d} s+I_{i},
\end{aligned}
$$

$$
\begin{aligned}
\dot{y}_{i}(t)= & -\dot{a}_{i}(t) y_{i}(t)+\sum_{j=1}^{n} \dot{b}_{i j}(t) \gamma_{j}(t)+\sum_{j=1}^{n} \dot{c}_{i j}(t) \bar{\gamma}_{j}\left(t-\tau_{j}(t)\right) \\
& +\sum_{j=1}^{n} \dot{d}_{i j}(t) \int_{t-\delta_{j}(t)}^{t} \gamma_{j}(s) \mathrm{d} s+I_{i}+K_{i}(t) .
\end{aligned}
$$

If the assumptions $\left(H_{1}\right)$ and $\left(H_{2}\right)$ hold, then, according to Definition 1, we know that $x_{i}(t)$ and $y_{i}(t)$ are the Filippov sense solutions of the drive system (1) and the response system (8), respectively, where $i \in I$.

Let $e_{i}(t)=y_{i}(t)-x_{i}(t), i \in I$, be the system of error. From the systems (13) and (14), we get the error system as follows:

$$
\begin{aligned}
\dot{e}_{i}(t)= & -\left(\grave{a}_{i}(t) y_{i}(t)-\widetilde{a}_{i}(t) x_{i}(t)\right)+\sum_{j=1}^{n}\left(\grave{b}_{i j}(t) \gamma_{j}(t)(t) \gamma_{j}(t)-\widetilde{b}_{i j}(t) \eta_{j}(t)\right) \\
& +\sum_{j=1}^{n}\left(\grave{c}_{i j}(t) \gamma_{j}(t)(t) \bar{\gamma}_{j}\left(t-\tau_{j}(t)\right)-\widetilde{c}_{i j}(t) \bar{\eta}_{j}\left(t-\tau_{j}(t)\right)\right) \\
& +\sum_{j=1}^{n} \grave{d}_{i j}(t) \gamma_{j}(t)(t) \int_{t-\delta_{j}(t)}^{t} \gamma_{j}(s) \mathrm{d} s-\sum_{j=1}^{n} \widetilde{d}_{i j}(t) \int_{t-\delta_{j}(t)}^{t} \eta_{j}(s) \mathrm{d} s+K_{i}(t), \quad i \in I .
\end{aligned}
$$

Definition 2. The drive-response systems (1) and (8) are said to reach the fixed-time synchronization, if, for any initial condition, there exist a fixed-time $T_{\max }$ and a settling time function $T(e(s))$ such that $\lim _{t \longrightarrow T(e(s))}\|e(t)\|=0$, and $\|e(t)\|=0, \forall t \geq T(e(s))$, where $T(e(s)) \leq T_{\max }$, and $e(s)=$ $\varphi(s)-\phi(s) \in R^{n} \quad$ and $\quad e(s)=\left(\varphi_{1}(s)-\phi_{1}(s), \varphi_{2}(s)-\phi_{2}\right.$ $\left.(s), \ldots, \varphi_{n}(s)-\phi_{n}(s)\right), s \in[-r, 0]$.

Besides, the following lemmas are necessary to derive the main results.

Lemma 1 (see [35]). For any constant vector $\zeta \in R^{n}$ and $0<r<l$, the two following inequalities hold:

$$
\left(\sum_{j=1}^{n}\left|\zeta_{j}\right|^{l}\right)^{1 / l} \leq\left(\sum_{j=1}^{n}\left|\zeta_{j}\right|^{r}\right)^{1 / r},\left(\frac{1}{n} \sum_{j=1}^{n}\left|\zeta_{j}\right|^{l}\right)^{1 / l} \geq\left(\frac{1}{n} \sum_{j=1}^{n}\left|\zeta_{j}\right|^{r}\right)^{1 / r} .
$$

Lemma 2 (see [46]). Suppose that there exists a continuous and radially unbounded function $V: R^{n} \longrightarrow R_{+} \bigcup\{0\}$ such that any solution $e(t)$ of system (15) satisfies the inequality

$$
\dot{V}(e(t)) \leq \lambda V(e(t))-\mu(V(e(t)))^{\gamma+\operatorname{sign}(V(e(t))-1)},
$$

in which $\lambda<\mu, \mu>0$, and $1<\gamma<2$; then the origin of system (15) is globally fixed-time stable. In addition, for any initial state $e_{0}$ of system (15), the settling time is estimated as

$$
T= \begin{cases}\frac{1}{\lambda(2-\gamma)} \ln \frac{\mu}{\mu-\lambda}+\frac{1}{\gamma(\mu-\lambda)}, & \text { if } \lambda>0, \\ \frac{1}{\mu(2-\gamma)}+\frac{1}{\mu \gamma}, \quad \text { if } \lambda=0, & \\ \frac{1}{\lambda(2-\gamma)} \ln \frac{\mu}{\mu-\lambda}+\frac{1}{\gamma \lambda} \ln \frac{\mu}{\mu-\lambda}, & \text { if } \lambda<0 .\end{cases}
$$

Lemma 3 (see [47]). If $V(\cdot): R^{n} \longrightarrow R$ is continuous and radially unbounded and it satisfies

(i) $V(e(t))=0 \Longleftrightarrow e(t)=0$

(ii) for any nonzero solution $e(t)$ of the system (15), there exist $\alpha, \beta>0,0 \leq \xi<1$ and $\eta>1$ such that

$$
\dot{V}(e(t)) \leq-\alpha V^{\xi}(e(t))-\beta V^{\eta}(e(t)),
$$

then the origin of system (15) is fixed-time stable, and

$$
T=\frac{1}{\alpha(1-\xi)}\left(\frac{\alpha}{\beta}\right)^{(1-\xi) /(\eta-\xi)}+\frac{1}{\beta(\eta-1)}\left(\frac{\alpha}{\beta}\right)^{(1-\eta) /(\eta-\xi)} .
$$

Lemma 4 (see [43]). For $i \in I$, the following inequality holds: 


$$
-\operatorname{sign}\left(y_{i}(t)-x_{i}(t)\right)\left(\grave{a}_{i}(t) y_{i}(t)-\tilde{a}_{i}(t) x_{i}(t)\right) \leq-\underline{a}_{i}\left|e_{i}(t)\right|+\left|a_{i}^{*}-a_{i}^{* *}\right| T_{i} \text {. }
$$

\section{Main Results}

In this section, we will derive some criteria to ensure the fixed-time synchronization for the drive-response systems (1) and (8) by designing a new nonlinear state-feedback controller and a novel switching state-feedback controller.

Firstly, the fixed-time synchronization based on a statefeedback controller will be considered.
3.1. Fixed-Time Synchronization under Nonlinear StateFeedback Controller. To obtain fixed-time synchronization, the nonlinear state-feedback controller in the response system (8) is designed as follows:

$$
K_{i}(t)=\bar{\alpha}_{i} e_{i}(t)-\operatorname{sign}\left(e_{i}(t)\right)\left(\beta_{i}+\xi_{i}\left|e_{i}(t)\right|^{2 \gamma+2 \operatorname{sign}\left((1 / 2)\|e(t)\|_{2}^{2}-1\right)-1}+\sum_{j=1}^{n} \bar{c}_{i j} Z_{j}\left|e_{j}\left(t-\tau_{j}(t)\right)\right|\right)
$$

where $\bar{\alpha}_{i}, \bar{\beta}_{i}, \xi_{i}, \gamma$ are control parameters, $\bar{\alpha}_{i}$ denotes the control gains, $\bar{\beta}_{i}>0, \xi_{i}>0, \quad(3 / 2)<\gamma<2$, and $\|e(t)\|_{2}=\left(\sum_{i=1}^{n}\left|e_{i}(t)\right|^{2}\right)^{1 / 2}$, for $i \in I$.

Remark 1. In papers [27, 28, 47-50], the controllers are similar to $u_{i}(t)=-\eta_{i} e_{i}(t)-\operatorname{sign}\left(e_{i}(t)\right)\left(\gamma_{i}+k_{1}\right.$ $\left.\left|e_{i}(t)\right|^{\beta_{1}}+k_{2}\left|e_{i}(t)\right|^{\beta_{2}}\right)-\delta_{i} \operatorname{sign}\left(e_{i}(t)\right)\left|e_{i}\left(t-\tau_{i}(t)\right)\right|$ in [48], where the index of one term is $0<\beta_{2}<1$, and the other one is $\beta_{1}>1$. However, in controller (22), the index term only has one term $2 \gamma+2 \operatorname{sign}(V(t)-1)-1$; it is a function of $V(t)$, not a constant, which means that the controller in this paper is more energy-saving.

For convenience, we assume that $\alpha_{i}=-\bar{\alpha}_{i}$ $-\underline{a}_{i}+(1 / 2) \sum_{j=1}^{n} \bar{b}_{i j} L_{j}+(1 / 2) \sum_{j=1}^{n} \bar{b}_{j i} L_{i}, i \in I$, and $\beta_{i}=\bar{\beta}_{i}-$ $\left|a_{i}^{*}-a_{i}^{* *}\right| T_{i}-\sum_{j=1}^{n} \bar{b}_{j i}\left(N_{j}+2 S_{j}\right)-\sum_{j=1}^{n} \bar{c}_{i j}\left(H_{j}+2 M_{j}\right)-2$ $\sum_{j=1}^{n} \bar{d}_{i j} S_{j} \delta_{j}, i \in I$.

Theorem 1. Suppose that $\left(H_{1}\right)-\left(H_{3}\right)$ hold; if $\beta_{i} \geq 0, i \in I$, then, under controller (22), the following statements hold:

Case (I): if $\lambda<0$, the drive-response systems (1) and (8) are synchronized in a fixed time and the settling time of fixed-time synchronization is estimated by

$$
T \leq \frac{1}{\lambda(2-\gamma)} \ln \frac{\xi 2^{\gamma-1}}{\xi 2^{\gamma-1}-\lambda}+\frac{1}{\lambda \gamma} \ln \frac{\xi n^{-\gamma} 2^{\gamma+1}}{\xi n^{-\gamma} 2^{\gamma+1}-\lambda}
$$

Case (II): if $\lambda=0$, the drive-response systems (1) and (8) are synchronized in a fixed time and the settling time of fixed-time synchronization is estimated by

$$
T \leq \frac{1}{\xi 2^{\gamma-1}(2-\gamma)}+\frac{1}{\xi n^{-\gamma} 2^{\gamma+1} \gamma} .
$$

Case (III): if $\lambda>0$, the drive-response systems (1) and (8) are synchronized in a fixed time and the settling time of fixed-time synchronization is estimated by

$$
T \leq \frac{1}{\lambda(2-\gamma)} \ln \frac{\xi 2^{\gamma-1}}{\xi 2^{\gamma-1}-\lambda}+\frac{1}{\gamma\left(\xi n^{-\gamma} 2^{\gamma+1}-\lambda\right)},
$$

$$
\text { where } \lambda=\max _{i \in I}\left\{2 \alpha_{i}\right\}, \xi=\min _{i \in I}\left\{\xi_{i}\right\} \text {. }
$$

Proof. Construct a Lyapunov function according to the following form:

$$
V(t)=\frac{1}{2} \sum_{i=1}^{n}\left|e_{i}(t)\right|^{2}
$$

Calculating the upper right derivative of $V(t)$ along the solution trajectories of the error system (15), one can get

$$
\begin{aligned}
D^{+} V(t)= & \sum_{i=1}^{n}\left|e_{i}(t)\right| \operatorname{sign}\left(e_{i}(t)\right) \dot{e}_{i}(t) \\
= & \sum_{i=1}^{n}\left|e_{i}(t)\right| \operatorname{sign}\left(e_{i}(t)\right)\left[-\left(\dot{a}_{i}(t) y_{i}(t)-\tilde{a}_{i}(t) x_{i}(t)\right)\right. \\
& +\sum_{j=1}^{n}\left(b_{i j}^{\prime}(t) \gamma_{j}(t)-\tilde{b}_{i j}(t) \eta_{j}(t)\right) \\
& +\sum_{j=1}^{n}\left(c_{i j}^{\prime}(t) \bar{\gamma}_{j}\left(t-\tau_{j}(t)\right)-\tilde{c}_{i j}(t) \bar{\eta}_{j}\left(t-\tau_{j}(t)\right)\right) \\
& +\sum_{j=1}^{n} d_{i j}^{\prime}(t) \int_{t-\delta_{j}(t)}^{t} \gamma_{j}(s) \mathrm{d} s \\
& \left.-\sum_{j=1}^{n} \widetilde{d}_{i j}(t) \int_{t-\delta_{j}(t)}^{t} \eta_{j}(s) \mathrm{d} s+K_{i}(t)\right] .
\end{aligned}
$$

Under $\left(\mathrm{H}_{2}\right)-\left(\mathrm{H}_{3}\right)$, for $i \in I$, the following inequalities can be obtained:

$$
\sum_{j=1}^{n}\left(b_{i j}^{\prime}(t) \gamma_{j}(t)-\widetilde{b}_{i j}(t) \eta_{j}(t)\right) \leq \sum_{j=1}^{n}\left[\bar{b}_{i j} L_{j}\left|e_{j}(t)\right|+\bar{b}_{i j}\left(N_{j}+2 S_{j}\right)\right],
$$




$$
\sum_{j=1}^{n}\left(\grave{c}_{i j}(t) \bar{\gamma}_{j}\left(t-\tau_{j}(t)\right)-\widetilde{c}_{i j}(t) \bar{\eta}_{j}\left(t-\tau_{j}(t)\right)\right) \leq \sum_{j=1}^{n}\left[\bar{c}_{i j} Z_{j}\left|e_{j}\left(t-\tau_{j}(t)\right)\right|+\bar{c}_{i j}\left(H_{j}+2 M_{j}\right)\right]
$$

By applying $\left(H_{3}\right)$, one can get

$$
\sum_{j=1}^{n} \grave{d}_{i j}(t) \int_{t-\delta_{j}(t)}^{t} \gamma_{j}(s) \mathrm{d} s-\sum_{j=1}^{n} \tilde{d}_{i j}(t) \int_{t-\delta_{j}(t)}^{t} \eta_{j}(s) \mathrm{d} s \leq 2 \sum_{j=1}^{n} \bar{d}_{i j} s_{j} \delta_{j}, \quad i \in I .
$$

Substituting (28), (29), and (30) into (27) and by using Lemma 4, the following inequality can be deduced:

$$
\begin{aligned}
D^{+} V(t) \leq & \sum_{i=1}^{n}\left[-\underline{a}_{i}\left|e_{i}(t)\right|^{2}+\left|e_{i}(t)\right|\left|a_{i}^{*}-a_{i}^{* *}\right| T_{i}\right. \\
& +\sum_{j=1}^{n}\left|e_{i}(t)\right|\left(\bar{b}_{i j} L_{j}\left|e_{j}(t)\right|+\bar{b}_{i j}\left(N_{j}+2 S_{j}\right)\right) \\
& +\sum_{j=1}^{n}\left|e_{i}(t)\right|\left(\bar{c}_{i j} Z_{j}\left|e_{j}\left(t-\tau_{j}(t)\right)\right|+\bar{c}_{i j}\left(H_{j}+2 M_{j}\right)\right) \\
& \left.+2 \sum_{j=1}^{n} \bar{d}_{i j} S_{j} \delta_{j}\left|e_{i}(t)\right|+\operatorname{sign}\left(e_{i}(t)\right)\left|e_{i}(t)\right| K_{i}(t)\right] .
\end{aligned}
$$

According to the elementary inequality $\left|e_{i}(t)\right|\left|e_{j}(t)\right| \leq(1 / 2)\left(\left|e_{i}(t)\right|^{2}+\left|e_{j}(t)\right|^{2}\right)$, one can obtain

$$
\begin{aligned}
D^{+} V(t) \leq & \sum_{i=1}^{n}\left[-\underline{a}_{i}\left|e_{i}(t)\right|^{2}+\left|e_{i}(t)\right|\left|a_{i}^{*}-a_{i}^{* *}\right| T_{i}\right. \\
& +\frac{1}{2} \sum_{j=1}^{n}\left|e_{i}(t)\right|^{2} \bar{b}_{i j} L_{j}+\frac{1}{2} \sum_{j=1}^{n}\left|e_{j}(t)\right|^{2} \bar{b}_{i j} L_{j} \\
& +\sum_{j=1}^{n}\left|e_{i}(t)\right| \bar{b}_{i j}\left(N_{j}+2 S_{j}\right)+\sum_{j=1}^{n}\left|e_{i}(t)\right| \bar{c}_{i j} Z_{j}\left|e_{j}\left(t-\tau_{j}(t)\right)\right| \\
& \left.\left.+\sum_{j=1}^{n}\left|e_{i}(t)\right| \bar{c}_{i}\left(H_{j}+2 M_{j}\right)\right)+2 \sum_{j=1}^{n} \bar{d}_{i j} S_{j} \delta_{j}\left|e_{i}(t)\right|+\operatorname{sign}\left(e_{i}(t)\right)\left|e_{i}(t)\right| K_{i}(t)\right] \\
= & \sum_{i=1}^{n}\left[\left|e_{i}(t)\right|^{2}\left(-\underline{a}_{i}+\frac{1}{2} \sum_{j=1}^{n} \bar{b}_{i j} L_{j}+\frac{1}{2} \sum_{j=1}^{n} \bar{b}_{j i} L_{i}\right)\right. \\
& +\left|e_{i}(t)\right|\left(\left|a_{i}^{*}-a_{i}^{* *}\right| T_{i}+\sum_{j=1}^{n} \bar{b}_{i j}\left(N_{j}+2 S_{j}\right)+\sum_{j=1}^{n} \bar{c}_{i j}\left(H_{j}+2 M_{j}\right)+2 \sum_{j=1}^{n} \bar{d}_{i j} S_{j} \delta_{j}\right) \\
& +\sum_{j=1}^{n}\left|e_{i}(t)\right| \bar{c}_{i j} Z_{j}\left|e_{i}\left(t-\tau_{j}(t)\right)\right| \\
& -\sum_{j=1}^{n}\left|e_{i}(t)\right| \bar{c}_{i j} Z_{j}\left|e_{j}\left(t-\tau_{j}(t)\right)\right|
\end{aligned}
$$




$$
\begin{aligned}
& \left.-\left|e_{i}(t)\right|^{2} \bar{\alpha}_{i}-\bar{\beta}_{i}\left|e_{i}(t)\right|-\xi_{i}\left|e_{i}(t)\right|^{2 \gamma+2 \operatorname{sign}(V(t)-1)}\right] \\
\leq & \sum_{i=1}^{n}\left(\alpha_{i}\left|e_{i}(t)\right|^{2}-\beta_{i}\left|e_{i}(t)\right|-\xi_{i}\left(\left|e_{i}(t)\right|^{2}\right)^{\gamma+\operatorname{sign}(V(t)-1)}\right) \\
\leq & \sum_{i=1}^{n}\left(\alpha_{i}\left|e_{i}(t)\right|^{2}-\xi_{i}\left(\left|e_{i}(t)\right|^{2}\right)^{\gamma+\operatorname{sign}(V(t)-1)}\right) \\
\leq & \lambda V(t)-\xi \sum_{i=1}^{n}\left(\left|e_{i}(t)\right|^{2}\right)^{\gamma+\operatorname{sign}(V(t)-1)} .
\end{aligned}
$$

Next, based on Lemma 1, we will deal with $-\xi \sum_{i=1}^{n}\left(\left|e_{i}(t)\right|^{2}\right)^{\gamma+\operatorname{sign}(V(t)-1)}$. Meanwhile the following inequalities can be deduced:

Case (I): when $0 \leq V(t)<1$, one can obtain

$$
-\xi \sum_{i=1}^{n}\left(\left|e_{i}(t)\right|^{2}\right)^{\gamma+\operatorname{sign}(V(t)-1)}=-\xi \sum_{i=1}^{n}\left(\left|e_{i}(t)\right|^{2}\right)^{\gamma-1} \text {. }
$$

Combining Lemma 1 and $0<\gamma-1<1$, one can get

$$
-\xi \sum_{i=1}^{n}\left(\left|e_{i}(t)\right|^{2}\right)^{\gamma-1} \leq-\xi 2^{\gamma-1}(V(t))^{\gamma-1}
$$

Case (II): when $V(t) \geq 1$, one will have

$$
-\xi \sum_{i=1}^{n}\left(\left|e_{i}(t)\right|^{2}\right)^{\gamma+\operatorname{sign}(V(t)-1)}=-\xi \sum_{i=1}^{n}\left(\left|e_{i}(t)\right|^{2}\right)^{\gamma+1} \text {. }
$$

Combining Lemma 1 and $\gamma+1>1$, one can obtain

$$
-\xi \sum_{i=1}^{n}\left(\left|e_{i}(t)\right|^{2}\right)^{\gamma+1} \leq-\xi n^{-\gamma} 2^{\gamma+1}(V(t))^{\gamma+1} .
$$

From equations (34) and (36), the following inequality can be deduced:

$$
\left.D^{+} V(t)\right) \leq\left\{\begin{array}{l}
\lambda V(t)-\xi n^{-\gamma} 2^{\gamma+1}(V(t))^{\gamma+1}, \quad V(t) \geq 1, \\
\lambda V(t)-\xi 2^{\gamma-1}(V(t))^{\gamma-1}, \quad 0 \leq V(t)<1,
\end{array}\right.
$$

where $\lambda<\min \left\{\xi n^{-\gamma} 2^{\gamma+1}, \xi 2^{\gamma-1}\right\}$.

From Lemma 2, the drive-response systems (1) and (8) achieve synchronization in a fixed time. And the settling time can be given as follows.

If $\lambda<0$, the settling time of fixed-time synchronization is estimated by

$$
T \leq \frac{1}{\lambda(2-\gamma)} \ln \frac{\xi 2^{\gamma-1}}{\xi 2^{\gamma-1}-\lambda}+\frac{1}{\lambda \gamma} \ln \frac{\xi n^{-\gamma} 2^{\gamma+1}}{\xi n^{-\gamma} 2^{\gamma+1}-\lambda},
$$

if $\lambda=0$, the settling time is estimated by

$$
T \leq \frac{1}{\xi 2^{\gamma-1}(2-\gamma)}+\frac{1}{\xi n^{-\gamma} 2^{\gamma+1} \gamma},
$$

and if $\lambda>0$, the settling time is estimated by

$$
T \leq \frac{1}{\lambda(2-\gamma)} \ln \frac{\xi 2^{\gamma-1}}{\xi 2^{\gamma-1}-\lambda}+\frac{1}{\gamma\left(\xi n^{-\gamma} 2^{\gamma+1}-\lambda\right)} .
$$

The proof of Theorem 1 is completed.

Remark 2. To avoid the chattering problem, the sign function in the controllers of $[49,51]$ was replaced by the continuous function tanh. Based on [49, 51], the sign function in controller (22) is replaced by the continuous function tanh to weaken or eliminate chattering phenomenon. For example, controller (22) can be modified as follows:

$$
\breve{K}_{i}(t)=-\bar{\alpha}_{i} e_{i}(t)-\tanh \left(e_{i}(t)\right)\left(\bar{\beta}_{i}+\xi_{i}\left|e_{i}(t)\right|^{2 \gamma+2 \operatorname{sign}\left((1 / 2)\|e(t)\|_{2}^{2}-1\right)-1}+\sum_{j=1}^{n} \bar{c}_{i j} Z_{j}\left|e_{j}\left(t-\tau_{j}(t)\right)\right|\right)
$$

where the control parameters $\bar{\alpha}_{i}, \bar{\beta}_{i}, \xi_{i}$, and $\gamma$ are also defined the same as in equation (22), for $i \in I$.
According to Theorem 1, the following corollary can be obtained. 
Corollary 1. Suppose that $\left(H_{1}\right)-\left(H_{3}\right)$ hold; then the driveresponse systems (1) and (8) reach fixed-time synchronization under the state-feedback controller (41) if $\alpha_{i}>0\left(\alpha_{i}=\right.$ $\left.0, \alpha_{i}<0\right)$ and $\beta_{i} \geq 0, i \in I$. Moreover, the settling time is defined the same as in Theorem 1, respectively.
In controller (22), after simple calculation, it is easy to get $0<2 \gamma+2 \operatorname{sign}(V(t)-1)-1<5$. When the power exponent $2 \gamma+2 \operatorname{sign}(V(t)-1)-1$ is replaced by a constant $\rho-1$ and $2<\rho<5$, then controller (22) is modified as follows:

$$
\widehat{K}_{i}(t)=-\bar{\alpha}_{i} e_{i}(t)-\operatorname{sign}\left(e_{i}(t)\right)\left(\bar{\beta}_{i}+\xi_{i}\left|e_{i}(t)\right|^{\rho-1}+\sum_{j=1}^{n} \bar{c}_{i j} Z_{j}\left|e_{j}\left(t-\tau_{j}(t)\right)\right|\right),
$$

where, for $i \in I$, the control parameters $\bar{\alpha}_{i}, \bar{\beta}_{i}$, and $\xi_{i}$ are defined the same as in (22), the index $\rho$ is a constant, and $2<\rho<5$. Let $\beta^{*}=\min _{i \in I}\left\{\beta_{i}\right\}$.

Based on Lemmas 1 and 3 and equation (32), we can get the following conclusion.

Corollary 2. Suppose that $\left(H_{1}\right)-\left(H_{3}\right)$ hold. Then, the drive-response systems (1) and (8) will reach synchronization in a fixed time under controller (42) if for $i \in I \alpha_{i}=0$ and $\beta_{i}>0$. In addition, the settling time is estimated as

$$
T \leq \frac{\sqrt{2}}{\beta^{*}}\left(\frac{2^{(1-\rho / 2)} \beta^{*}}{\xi n^{(2-\rho / 2)}}\right)^{(1 / \rho-1)}+\frac{2^{(2-\rho / 2)}}{\xi n^{(2-\rho / 2)}(\rho-2)}\left(\frac{2^{(1-\rho / 2)} \beta^{*}}{\xi n^{(2-\rho / 2)}}\right)^{(2-\rho / \rho-1)} .
$$

Remark 3. In paper [44], the authors employing the traditional fixed-time stability theory in [24] obtained the settling time $T$. Meanwhile, in Corollary 2, the settling time $T$ of fixed-time synchronization is given by applying Lemma 3 . Based on literature [47], one can know that, under the same conditions, the settling time $T$ of Corollary 2 in this paper is smaller than that in [44]. That is to say, the settling time $T$ in this paper is closer to the real synchronization time.

Similar to controller (41), the discontinuous sign function in controller (42) is replaced by the continuous function tanh. We can deduce the following no-sign function controller:

$$
\underline{K}_{i}(t)=-\bar{\alpha}_{i} e_{i}(t)-\tanh \left(e_{i}(t)\right)\left(\bar{\beta}_{i}+\xi_{i}\left|e_{i}(t)\right|^{\rho-1}+\sum_{j=1}^{n} \bar{c}_{i j} Z_{j}\left|e_{j}\left(t-\tau_{j}(t)\right)\right|\right),
$$

where the parameters are the same as in controller (22).

Corollary 3. Suppose that $\left(H_{1}\right)-\left(H_{3}\right)$ hold. Then, the driveresponse systems (1) and (8) will reach synchronization in a fixed time under controller (44) if for $i \in I \alpha_{i}=0$ and $\beta_{i}>0$. Meanwhile, the settling time $T$ is defined the same as in Corollary 2.

Remark 4. It is noted that there is a sign function in the controller of $[27,28,44,47,48]$, which will inevitably lead to the chattering phenomenon, while in controller (44) there is not. Therefore, the conclusion of Corollary 3 has better practicability.

Remark 5. In papers [41, 42], the synchronization for a class of memristor-based neural networks with discontinuous neuron activations and mixed time-varying delays was investigated, while the time of synchronization is infinite, not within a fixed time. Therefore, this paper improves some previous conclusions.

Secondly, the fixed-time synchronization based on a switching state-feedback controller will be focused on.

\subsection{Fixed-Time Synchronization under the Switching State-} Feedback Controller. To obtain the response system (8), which is synchronized to the drive system (1), the following switching state-feedback controller is designed as

$$
\bar{U}_{i}(t)=\left\{\begin{array}{l}
-\bar{\omega}_{i} e_{i}(t)-q_{i} \frac{e_{i}(t)}{\|e(t)\|_{1}}-\sum_{j=1}^{n} \bar{c}_{i j} Z_{j} \frac{e_{i}(t)}{\|e(t)\|_{1}}\left|e_{j}\left(t-\tau_{j}(t)\right)\right|, \\
-\iota_{i} e_{i}(t)\left|e_{i}(t)\right|^{\gamma+\operatorname{sign}\left(\|e(t)\|_{1}-1\right)-1}, \quad\|e(t)\|_{1} \neq 0, \\
0, \quad\|e(t)\|_{1}=0,
\end{array}\right.
$$

where the control parameters $\bar{\omega}_{i}$ refer to the control gains, $q_{i}$, $1<\gamma<2$, and $\lambda_{i}$ are positive constants, for $i \in I$; and $\|e(t)\|_{1}=\sum_{i=1}^{n}\left|e_{i}(t)\right|$.

Remark 6. Compared with the switching state-feedback controller in literature [52], the power exponent in equation (45) is a function of the system error state rather than a constant. Furthermore, there is no sign function in the control parameters coefficient in the above controller (45), which not only reduces or eliminates the chattering phenomenon but also saves energy. In addition, the switching state-feedback controller has another advantage lying in overcoming the uncertainty difference of Filippov solution and the influence of mixed time-varying delays. From a practical point of view, controller (45) has a stronger practicability. 
For the sake of convenience, $\omega_{i}$ and $\chi_{i}$ are denoted: $\omega_{i}=-\bar{\omega}_{i}-\underline{a}_{i}+\sum_{j=1}^{n} \bar{b}_{j i} L_{i}$, and $\chi_{i}=-\left|a_{i}^{*}-a_{i}^{* *}\right| T_{i}-\sum_{j=1}^{n} \bar{b}_{i j}$ $\left(N_{j}+2 S_{j}\right)-\sum_{j=1}^{n}\left[\bar{c}_{i j}\left(H_{j}+2 M_{j}\right)+2 \bar{d}_{i j} S_{j} \delta_{j}\right], i \in I$.

Theorem 2. Suppose that $\left(H_{1}\right)-\left(H_{3}\right)$ hold; if $q_{i}+\chi_{i} \geq 0, i \in I$, then, under controller (45), the following statements hold:

Case (I): if $\omega<0$, the drive-response systems (1) and (8) are synchronized in fixed time and the settling time is estimated by

$$
T \leq \frac{1}{\omega(2-\gamma)} \ln \frac{\curlywedge}{\curlywedge-\omega}+\frac{1}{\omega \gamma} \ln \frac{\measuredangle n^{-\gamma}}{\measuredangle n^{-\gamma}-\omega} .
$$

Case (II): if $\omega=0$, the drive-response systems (1) and (8) are synchronized in a fixed time and the settling time is estimated by

$$
T \leq \frac{1}{\curlywedge(2-\gamma)}+\frac{1}{\measuredangle n^{-\gamma} \gamma}
$$

Case (III): if $\omega>0$, the drive-response systems (1) and (8) are synchronized in a fixed time and the settling time is estimated by

$$
T \leq \frac{1}{\omega(2-\gamma)} \ln \frac{\iota}{\curlywedge-\omega}+\frac{1}{\omega \gamma} \ln \frac{<n^{-\gamma}}{\wedge n^{-\gamma}-\omega},
$$

where $\omega=\max _{i \in I}\left\{\omega_{i}\right\}$ and $\curlywedge=\min _{i \in I}\left\{\alpha_{i}\right\}$.

Proof. Construct a Lyapunov function as follows:

$$
V(t)=\sum_{i=1}^{n}\left|e_{i}(t)\right|
$$

Calculating the upper right derivative of $V(t)$ along the solution of the error system (15), one can get

$$
\begin{aligned}
D^{+} V(t)= & \sum_{i=1}^{n} \operatorname{sign}\left(e_{i}(t)\right) \dot{e}_{i}(t) \\
= & \sum_{i=1}^{n} \operatorname{sign}\left(e_{i}(t)\right)\left[-\left(\grave{a}_{i}(t) y_{i}(t)-\widetilde{a}_{i}(t) x_{i}(t)\right)\right. \\
& +\sum_{j=1}^{n}\left(\grave{b}_{i j}(t) \gamma_{j}(t)-\widetilde{b}_{i j}(t) \eta_{j}(t)\right) \\
& +\sum_{j=1}^{n}\left(\grave{c}_{i j}(t) \bar{\gamma}_{j}\left(t-\tau_{j}(t)\right)-\widetilde{c}_{i j}(t) \bar{\eta}_{j}\left(t-\tau_{j}(t)\right)\right) \\
& +\sum_{j=1}^{n} \grave{d}_{i j}(t) \int_{t-\delta_{j}(t)}^{t} \gamma_{j}(s) \mathrm{d} s \\
& \left.-\sum_{j=1}^{n} \widetilde{d}_{i j}(t) \int_{t-\delta_{j}(t)}^{t} \eta_{j}(s) \mathrm{d} s+\bar{U}_{i}(t)\right],
\end{aligned}
$$

and substituting equations (28), (29), and (30) into equation (50), with the help of Lemma 4 , one can obtain

$$
\begin{aligned}
D^{+} V(t) \leq & \sum_{i=1}^{n}\left[-\underline{a}_{i}\left|e_{i}\right|+\left|a_{i}^{*}-a_{i}^{* * *}\right| T_{i}\right. \\
& +\sum_{j=1}^{n}\left(\bar{b}_{i j} L_{j}\left|e_{j}(t)\right|+\bar{b}_{i j}\left(N_{j}+2 S_{j}\right)\right) \\
& +\sum_{j=1}^{n}\left(\bar{c}_{i j} Z_{j}\left|e_{j}\left(t-\tau_{j}(t)\right)\right|+\bar{c}_{i j}\left(H_{j}+2 M_{j}\right)\right) \\
& \left.+2 \sum_{j=1}^{n} \bar{d}_{i j} S_{j} \delta_{j}+\operatorname{sign}\left(e_{i}(t)\right) \bar{U}_{i}(t)\right] \\
\leq & \sum_{i=1}^{n}\left[\left|e_{i}(t)\right|\left(-\underline{a}_{i}+\sum_{j=1}^{n} \bar{b}_{j i} L_{i}\right)\right. \\
& +\sum_{j=1}^{n}\left(\bar{c}_{i j} Z_{j}\left|e_{j}\left(t-\tau_{j}(t)\right)\right|\right)+\sum_{j=1}^{n}\left(\left|a_{i}^{*}-a_{i}^{* *}\right| T_{i}\right) \\
& \left.+\bar{b}_{i j}\left(N_{j}+2 S_{j}\right)+\bar{c}_{i j}\left(H_{j}+2 M_{j}\right)+2 \bar{d}_{i j} S_{j} \delta_{j}\right)-\widetilde{\omega}_{i}\left|e_{i}(t)\right| \\
& \left.-\sum_{j=1}^{n} \bar{c}_{i j} Z_{j}\left|e_{j}\left(t-\tau_{j}(t)\right)\right|-\iota_{i}\left|e_{i}(t)\right|^{\gamma+\operatorname{sign}\left(\|e(t)\|_{1}-1\right)}-q_{i}\right] \\
\leq & \omega V(t)-<\sum_{i=1}^{n}\left|e_{i}(t)\right|^{\gamma+\operatorname{sign}\left(|e(t)|_{1}-1\right)} .
\end{aligned}
$$

Next, based on Lemma 1, we will deal with $-<\sum_{i=1}^{n}\left|e_{i}(t)\right|^{\gamma+\operatorname{sign}\left(\|e(t)\|_{1}-1\right)} ;$ simultaneously the following inequalities can be obtained:

Case (I): when $0 \leq V(t)<1$, one can get

$$
-<\sum_{i=1}^{n}\left|e_{i}(t)\right|^{\gamma+\operatorname{sign}\left(\|e(t)\|_{1}-1\right)}=-<\sum_{i=1}^{n}\left|e_{i}(t)\right|^{\gamma-1} \text {. }
$$

By applying Lemma 1 and $0<\gamma-1<1$, one can get

$$
-<\sum_{i=1}^{n}\left|e_{i}(t)\right|^{\gamma-1} \leq-<(V(t))^{\gamma-1} .
$$

Case (II): when $V(t) \geq 1$, one can have

$$
-\curlywedge \sum_{i=1}^{n}\left|e_{i}(t)\right|^{\gamma+\operatorname{sign}\left(\|e(t)\|_{1}-1\right)}=-\curlywedge \sum_{i=1}^{n}\left|e_{i}(t)\right|^{\gamma+1} .
$$

With the help of Lemma 1 and $\gamma+1>1$, one can obtain

$$
-<\sum_{i=1}^{n}\left|e_{i}(t)\right|^{\gamma+1} \leq-<n^{-\gamma}(V(t))^{\gamma+1} .
$$

From equations (53) and (55), the following inequality can be derived:

$$
\left.D^{+} V(t)\right) \leq \begin{cases}\omega V(t)-<n^{-\gamma}(V(t))^{\gamma+1}, & V(t) \geq 1, \\ \omega V(t)-\iota(V(t))^{\gamma-1}, & 0 \leq V(t)<1,\end{cases}
$$

where $\omega<\wedge n^{-\gamma}$. 
From Lemma 2, the drive-response systems (1) and (8) reach synchronization in a fixed time; and the settling time can be given as follows.

If $\omega<0$, the settling time is estimated by

$$
T \leq \frac{1}{\omega(2-\gamma)} \ln \frac{\iota}{\curlywedge-\omega}+\frac{1}{\omega \gamma} \ln \frac{<n^{-\gamma}}{\curlywedge n^{-\gamma}-\omega},
$$

if $\omega=0$, the settling time is estimated by

$$
T \leq \frac{1}{\wedge(2-\gamma)}+\frac{1}{\wedge n^{-\gamma} \gamma}
$$

and if $\omega>0$, the settling time is estimated by

$$
T \leq \frac{1}{\omega(2-\gamma)} \ln \frac{\iota}{\curlywedge-\omega}+\frac{1}{\omega \gamma} \ln \frac{\iota n^{-\gamma}}{\curlywedge n^{-\gamma}-\omega} .
$$

The proof of Theorem 2 is completed.

Next, we will consider a special case of the drive system (1) and the response system (8). When connection weight of the distributed time delay $d_{i j}(t)=0, i, j \in I$, the drive system (1) is reduced to the following form:

$$
\begin{aligned}
\dot{x}_{i}(t)= & -a_{i}\left(x_{i}(t)\right) x_{i}(t)+\sum_{j=1}^{n} b_{i j}\left(x_{i}(t)\right) f_{j}\left(x_{j}(t)\right)+\sum_{j=1}^{n} c_{i j}\left(x_{i}(t)\right) \\
& \times g_{j}\left(x_{j}\left(t-\tau_{j}(t)\right)\right)+I_{i} .
\end{aligned}
$$
form:

The response system (8) is degenerated to the following

$$
\begin{aligned}
\dot{y}_{i}(t)= & -a_{i}\left(y_{i}(t)\right) v_{i}(t)+\sum_{j=1}^{n} b_{i j}\left(y_{i}(t)\right) f_{j}\left(y_{j}(t)\right)+\sum_{j=1}^{n} c_{i j}\left(y_{i}(t)\right) \\
& \times g_{j}\left(y_{j}\left(t-\tau_{j}(t)\right)\right)+I_{i}+\bar{U}_{i}(t) .
\end{aligned}
$$

The control parameter $\chi_{i}$ becomes as follows:

$$
\tilde{\chi}_{i}=-\left|a_{i}^{*}-a_{i}^{* *}\right| T_{i}-\sum_{j=1}^{n} \bar{b}_{i j}\left(N_{j}+2 S_{j}\right)-\sum_{j=1}^{n}\left[\bar{c}_{i j}\left(H_{j}+2 M_{j}\right)\right], \quad i \in I .
$$

According to Theorem 2, we can obtain the following result.

Corollary 4. Suppose that $\left(H_{1}\right)-\left(H_{3}\right)$ hold; if $q_{i}+\sum_{i=1}^{n} \tilde{\chi}_{i} \geq 0$ and $\omega_{i}>0\left(\omega_{i}=0, \omega_{i}<0\right), i \in I$, then the drive-response systems (60) and (61) will reach fixed-time synchronization under controller (45); and the settling time is defined the same as that in Theorem 2, respectively.

Remark 7. In this paper, the neural network model includes memristor, mixed time-varying delay, and discontinuous activation function. In paper [53], the authors only focused on the discontinuous neuron activation function, but the effect of mixed time-varying delays on control synchronization was neglected; and, in paper [54], the effect of distributed delay on systems finite-time synchronization is not considered; in addition, the activation functions of neurons were continuous. Therefore, the mathematical model of the neural network of this paper is more general.
Based on controller (45), the following no-sign function switching state-feedback controller can be obtained:

$$
\widehat{U}_{i}(t)=\left\{\begin{array}{l}
-\bar{\omega}_{i} e_{i}(t)-q_{i} \frac{e_{i}(t)}{\|e(t)\|_{1}}-\sum_{j=1}^{n} \bar{c}_{i j} Z_{j} \frac{e_{i}(t)}{\|e(t)\|_{1}}\left|e_{j}\left(t-\tau_{j}(t)\right)\right|, \\
-\iota_{i} e_{i}(t)\left|e_{i}(t)\right|^{\gamma-1}, \quad\|e(t)\|_{1} \neq 0, \\
0, \quad\|e(t)\|_{1}=0,
\end{array}\right.
$$

where $1<\gamma<2$ and the other control parameters are the same as those in the switching state-feedback controller (45). Let $\widetilde{a}=\sum_{i=1}^{n}\left(q_{i}+\widetilde{\chi}_{i}\right)$.

With the help of equation (53) and by applying Lemmas 1 and 3 , the following result can be obtained.

Corollary 5. Suppose that $\left(H_{1}\right)-\left(H_{3}\right)$ hold. If the control parameters $q_{i}$ satisfy $q_{i}+\widetilde{\chi}_{i} \geq 0$ and $\omega_{i}=0$, then the drive- 
response systems (1) and (8) will reach fixed-time synchronization with the switching state-feedback controller (63). Moreover, the settling time is estimated by

$$
T \leq \frac{1}{\tilde{a}}\left(\frac{\widetilde{a} n^{\gamma}}{\curlywedge}\right)^{(1 / \gamma+1)}+\frac{n^{\gamma}}{\gamma \curlywedge}\left(\frac{\widetilde{a} n^{\gamma}}{\curlywedge}\right)^{\left(-\gamma n^{\gamma} / \curlywedge\right)} .
$$

In controller (45), we remove the term sign $\left(\|e(t)\|_{1}-1\right)$ in index term, and a switching state-feedback controller with no-sign function can be got. Afterwards, let $0<\gamma<1$; by using the common finite-time stability theory in [51], the following results can be obtained.

Corollary 6. Suppose that $\left(H_{1}\right)-\left(H_{3}\right)$ hold; if $q_{i}-\left|a_{i}^{*}-a_{i}^{* *}\right| T_{i}-2 \sum_{j=1}^{n}\left(\bar{b}_{i j} S_{j}+M_{j}+\bar{d}_{i j} S_{j} \delta_{j}\right) \geq 0$ and $\omega_{i}=0, i \in I$, under the modified controller (45), then the drive-response systems (1) and (8) are synchronized in a finite time and the settling time is estimated to be $T \leq\left(\|\varphi(0)-\phi(0)\|^{1-\gamma / \curlywedge}(1-\gamma)\right)$.

Next, we consider another special case of neural network mathematical model in this paper; that is, the neuron activation functions are continuous.

Corollary 7. Suppose that $\left(\mathrm{H}_{3}\right)-\left(\mathrm{H}_{4}\right)$ hold; if $q_{i}-\left|a_{i}^{*}-a_{i}^{* *}\right| T_{i}-2 \sum_{j=1}^{n}\left(\bar{b}_{i j} S_{j}+M_{j}+\bar{d}_{i j} S_{j} \delta_{j}\right) \geq 0 \quad$ and $\omega_{i}>0\left(\omega_{i}=0, \omega_{i}<0\right), i \in I$, under controller (45), then the drive-response systems (1) and (8) are synchronized in a fixed time and the settling time is defined the same as in Theorem 2, respectively.

Remark 8. In Theorem 2 and Corollary 6, the main differences in conditions $q_{i}-\left|a_{i}^{*}-a_{i}^{* *}\right| T_{i}-\sum_{j=1}^{n} \bar{b}_{i j}$ $\left(N_{j}+2 S_{j}\right)-\sum_{j=1}^{n}\left[\bar{c}_{i j}\left(H_{j}+2 M_{j}\right)+2 \bar{d}_{i j} S_{j} \delta_{j}\right] \geq 0 \quad$ and $\left.q_{i}-\left|a_{i}^{*}-a_{i}^{* *}\right| T_{i}-2 \sum_{j=1}^{n}\left(\bar{b}_{i j}\left(S_{j}\right)+M_{j}\right)+\bar{d}_{i j} S_{j} \delta_{j}\right) \geq 0$ are $N_{j}=H_{j}=0$; other parameters are the same. That is to say, controller (45) is suitable for both continuous and discontinuous neuron activation functions. Also, there is nosign function in the control parameters. Therefore, our method improves some previous results.

\section{Numerical Simulations}

In this section, two numerical examples are given to show the correctness and the effectiveness of the main results in this paper.

\subsection{Example of Fixed-Time Synchronization under Nonlinear State-Feedback Controller}

Example 1. The following 2-dimensional memristive neural networks with discontinuous neuron activation functions and mixed time-varying delays are considered as the drive system:

$$
\begin{aligned}
\dot{x}_{i}(t)= & -a_{i}\left(x_{i}(t)\right) x_{i}(t)+\sum_{j=1}^{2} b_{i j}\left(x_{j}(t)\right) f_{j}\left(x_{j}(t)\right)+\sum_{j=1}^{2} c_{i j}\left(x_{j}\left(t-\tau_{j}(t)\right)\right) \\
& \times g_{j}\left(x_{j}\left(t-\tau_{j}(t)\right)\right)+\sum_{j=1}^{2} d_{i j}\left(x_{j}(t)\right) \int_{t-\delta_{j}(t)}^{t} f_{j}\left(x_{j}(s)\right) \mathrm{d} s+I_{i},
\end{aligned}
$$

where $\left.\quad i=1,2, \quad f_{1}(u)\right)=f_{2} \quad(u)=1.21 \tanh (u)+\quad \tau_{1}(t)=\tau_{2}(t)=\left(e^{t} / 1+e^{t}\right), \delta_{1}(t)=\delta_{2}(t)=1$, and $I_{1}=I_{2}=$ $0.04 \operatorname{sign}(u), g_{1}(u)=g_{2} \quad(u)=1.1 \sin (u)+0.03 \operatorname{sign}(u), \quad 0$, and the values of the memristors are as follows: 


$$
\begin{aligned}
& a_{1}\left(x_{1}(t)\right)= \begin{cases}0.4, & \left|x_{1}(t)\right| \leq 1.1, \\
-0.32, & \left|x_{1}(t)\right|>1.1,\end{cases} \\
& a_{2}\left(x_{2}(t)\right)= \begin{cases}0.51, & \left|x_{2}(t)\right| \leq 1.1, \\
-0.2, & \left|x_{2}(t)\right|>1.1,\end{cases} \\
& b_{11}\left(x_{1}(t)\right)= \begin{cases}-1.2, & \left|x_{1}(t)\right| \leq 1.1, \\
-0.1, & \left|x_{1}(t)\right|>1.1,\end{cases} \\
& b_{12}\left(x_{1}(t)\right)= \begin{cases}0.9, & \left|x_{1}(t)\right| \leq 1.1, \\
-1.2, & \left|x_{1}(t)\right|>1.1,\end{cases} \\
& b_{21}\left(x_{2}(t)\right)= \begin{cases}1.4, & \left|x_{2}(t)\right| \leq 1.1, \\
-0.8, & \left|x_{2}(t)\right|>1.1,\end{cases} \\
& d_{22}\left(x_{2}(t)\right)= \begin{cases}-1.5, & \left|x_{2}(t)\right| \leq 1.1, \\
-0.4, & \left|x_{2}(t)\right|>1.1 .\end{cases} \\
& d_{22}\left(x_{2}(t)\right)= \begin{cases}-1, & \left|x_{2}(t)\right| \leq 1.1, \\
1.6, & \left|x_{2}(t)\right|>1.1,\end{cases} \\
& d_{12}\left(x_{1}(t)\right)= \begin{cases}-0.65, & \left|x_{1}(t)\right| \leq 1.1, \\
-0.5, & \left|x_{1}(t)\right|>1.1,\end{cases} \\
& c_{11}\left(x_{1}\left(x_{1}(t)\right)= \begin{cases}-1.1, & \left|x_{1}(t)\right| \leq 1.1, \\
-0.6, & \left|x_{1}(t)\right| \leq 1.1,\end{cases} \right.
\end{aligned}
$$

The model (65) has chaotic attractor with the initial values $x_{1}(s)=-0.4$ and $x_{2}(s)=0.6$ for $s \in[-1,0]$ which can be seen in Figure 1; and the state trajectories of system (65) with initial conditions $x_{1}(s)=-0.4, x_{2}=0.6$ are described in Figures 2 and 3.

The response system is given by

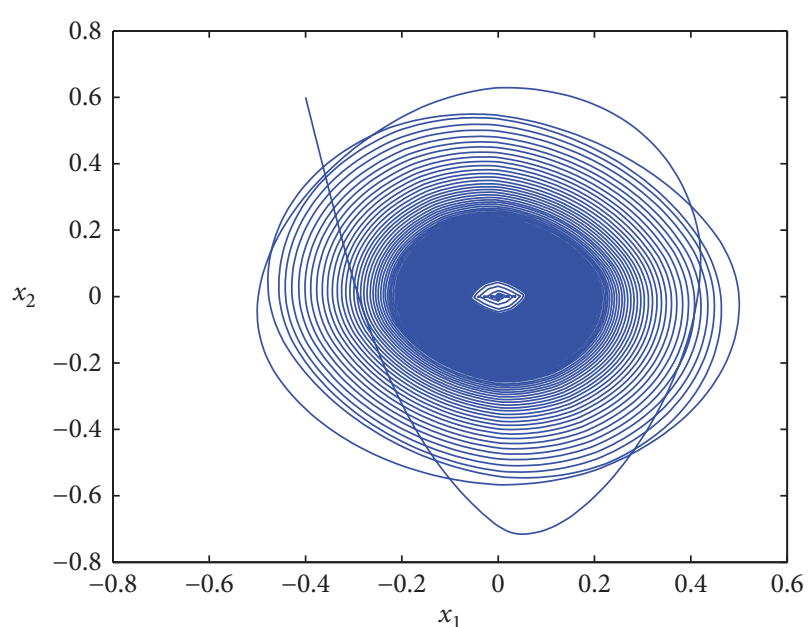

FIgURE 1: The chaotic attractor of system (65) with the initial values $x_{1}(s)=-0.4$ and $x_{2}(s)=0.6$, for $s \in[-1,0]$.

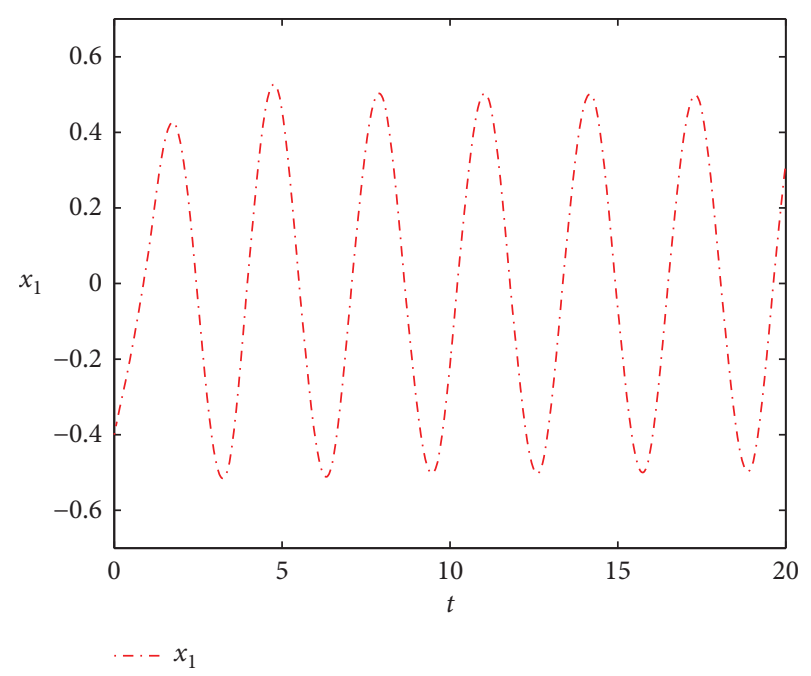

FIgURe 2: The dynamical behaviors for $x_{1}(t)$ of system (65) with the initial value $x_{1}(s)=-0.4$, for $s \in[-1,0]$.

$$
\begin{aligned}
\dot{y}_{i}(t)= & -a_{i}\left(y_{i}(t)\right) y_{i}(t)+\sum_{j=1}^{2} b_{i j}\left(y_{j}(t)\right) f_{j}\left(y_{j}(t)\right) \\
& +\sum_{j=1}^{2} c_{i j}\left(y_{j}\left(t-\tau_{j}(t)\right)\right) \\
& \times g_{j}\left(y_{j}\left(t-\tau_{j}(t)\right)\right)+\sum_{j=1}^{2} d_{i j}\left(y_{j}(t)\right) \\
& \cdot \int_{t-\delta_{j}(t)}^{t} f_{j}\left(y_{j}(s)\right) \mathrm{d} s+I_{i}+K_{i}(t),
\end{aligned}
$$

where the parameters $a_{i}(\cdot), b_{i j}(\cdot), c_{i j}(\cdot), d_{i j}(\cdot), f_{j}(\cdot), \tau_{j}(\cdot)$, and $\delta_{j}(t)$ are defined the same as those in system (67). Through simple calculation, we know that $L_{1}=L_{2}=1.21$, $N_{1}=N_{2}=0.04, \quad Z_{1}=Z_{2}=1.1, \quad H_{1}=H_{2}=0.03$, $S_{1}=S_{2}=1.25$, and $M_{1}=M_{2}=1.13$. 


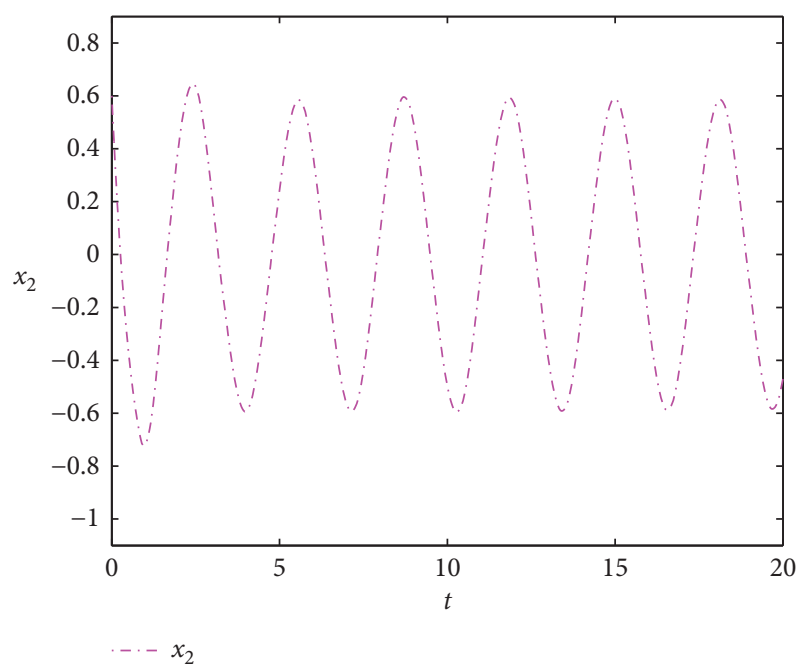

Figure 3: The dynamical behaviors for $x_{2}(t)$ of system (65) with the initial value $x_{2}(s)=0.6$, for $s \in[-1,0]$.

It is easy to check that the activation functions $f_{1}, f_{2}, g_{1}$, and $g_{2}$ satisfy assumptions $\left(H_{1}\right)-\left(H_{4}\right)$.

It is easy to get that $\underline{a}_{1}=-0.32, \underline{a}_{2}=-0.2, \bar{a}_{1}=0.4$, $\bar{a}_{2}=0.51, \bar{b}_{11}=1.2, \bar{b}_{12}=1.2, \bar{b}_{21}=1.4, \bar{b}_{22}=1.6, \bar{c}_{11}=1.1$, $\bar{c}_{12}=1.45, \bar{c}_{21}=1.6, \bar{c}_{22}=1.7$, and $\bar{d}_{11}=0.6, \bar{d}_{12}=0.5, \bar{d}_{21}=$ $1.4, \bar{d}_{22}=1.5$.

Take the control parameters $\bar{\beta}_{1}=17$ and $\bar{\beta}_{2}=23$. By simple calculation, $1.0145=\beta_{1}=\bar{\beta}_{1}-\left|a_{1}^{*}-a_{1}^{* * *}\right| T_{1}-$ $\sum_{j=1}^{2} \bar{b}_{j 1}\left(N_{j}+2 S_{j}\right)-\sum_{j=1}^{2} \bar{c}_{1 j}\left(H_{j}+2 M_{j}\right)-2 \sum_{j=1}^{2} \bar{d}_{1 j} S_{j} \delta_{j}$ $\geq 0$, and $0.3=\beta_{2}=\bar{\beta}_{2}-\left|a_{2}^{*}-a_{2}^{* *}\right| T_{2}-\sum_{j=1}^{2} \bar{b}_{j 2}\left(N_{j}+\right.$ $\left.2 S_{j}\right)-\sum_{j=1}^{2} \bar{c}_{2 j}\left(H_{j}+2 M_{j}\right)-2 \sum_{j=1}^{2} \bar{d}_{2 j} S_{j} \delta_{j} \geq 0$.

It can be seen from Figures 4 and 5 that when no controller is added to the response system (67), the state curves do not achieve fixed-time synchronization in Example 1.

According to the different controllers (26) and (41), numerical simulation of Example 1 will be divided into Case (A) and Case (B). Firstly, Case (A) will be simulated numerically as follows:

Case (A): under controller (26).

We take the control parameters $\xi=0.9$ and $\gamma=1.55$; and we will calculate parameter $\lambda$ about the control gain $\bar{\alpha}_{i}$ in three cases:

Case (I): $0.4=\lambda>0$.

When $\bar{\alpha}_{1}=3.245, \quad 0.1=\alpha_{1}=-\bar{\alpha}_{1}-\underline{a}_{1}+(1 / 2) \sum_{j=1}^{2}$ $\bar{b}_{1 j} L_{j}+(1 / 2) \sum_{j=1}^{2} \bar{b}_{j 1} L_{1}>0$; when $\bar{\alpha}_{2}=3.509,0.2=$ $\alpha_{2}=-\bar{\alpha}_{2}-\underline{a}_{2}+(1 / 2) \sum_{j=1}^{2} \bar{b}_{2 j} L_{j}+(1 / 2) \sum_{j=1}^{2} \bar{b}_{j 2} L_{2}$ $>0$; it can be found that all the conditions of Theorem 1 are satisfied, and the drive-response systems (1) and (8) reach fixed-time synchronization.

When $\lambda>0$, the fixed-time synchronization errors between the systems (65) and (67) are demonstrated in Figures 6-9, where 8 classes of different initial values are chosen for equations (65) and (67) by $x_{1}^{k}(s)=-0.4, x_{2}^{k}(s)=0.6, y_{1}^{k}(s)=-0.15+0.35 k$, and $y_{2}^{k}(s)=0.55-0.35 k, \quad$ for $\quad s \in[-1,0] \quad$ and

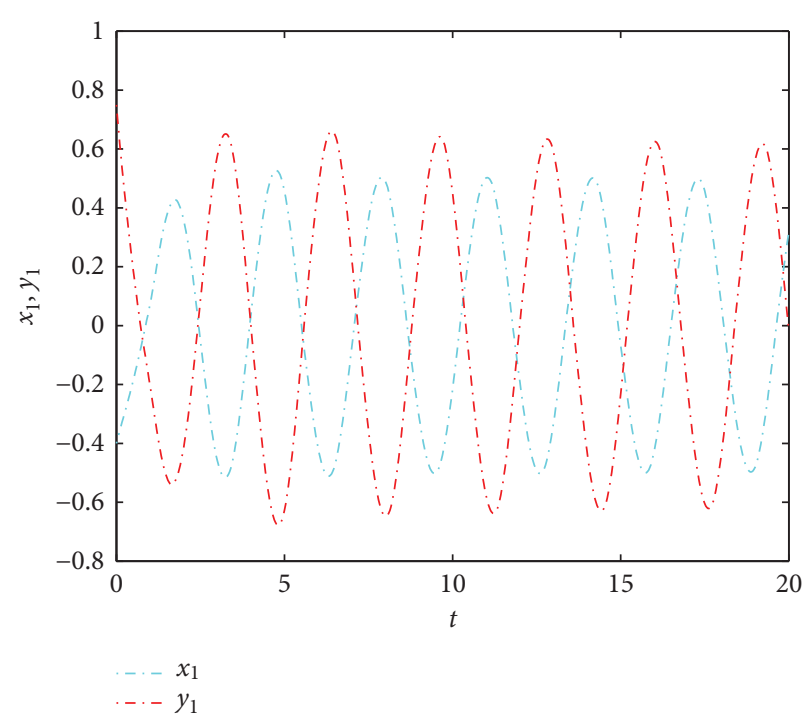

FIgURE 4: The state curves of $x_{1}(t)$ and $y_{1}(t)$ without control input in Example 1.

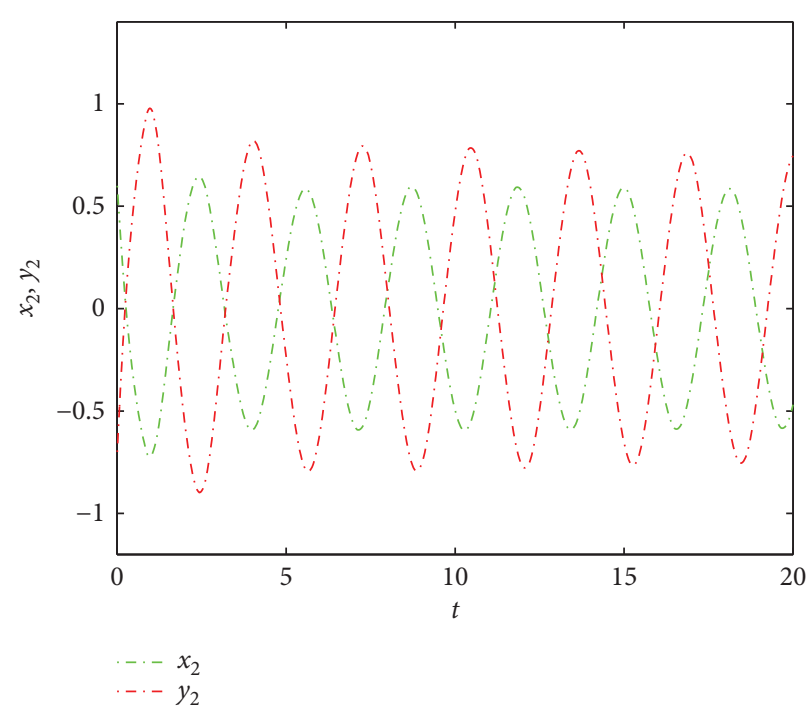

FIgURE 5: The state curves of $x_{2}(t)$ and $y_{2}(t)$ without control input in Example 1.

$k \in\{-4,-3,-2,-1,0,1,3\}$. Figures $6-9$ show that our results are effective, when $\lambda>0$. Through calculation, the settling time is estimated by $T \leq 2.4707$.

Case (II): $\lambda=0$.

When $\quad \bar{\alpha}_{1}=3.345, \quad 0=\alpha_{1}=-\bar{\alpha}_{1}-\underline{a}_{1}+(1 / 2) \sum_{j=1}^{2}$ $\bar{b}_{1 j} L_{j}+(1 / 2) \sum_{j=1}^{2} \bar{b}_{j 1} L_{1}$; when $\bar{\alpha}_{2}=3.709,0=\alpha_{2}=$ $-\bar{\alpha}_{2}-\underline{a}_{2}+(1 / 2) \sum_{j=1}^{2} \bar{b}_{2 j} L_{j}+(1 / 2) \sum_{j=1}^{2} \bar{b}_{j 2} L_{2}$, which implies that all the conditions of Theorem 1 are satisfied, and the drive-response systems (1) and (8) reach fixed-time synchronization.

When $\lambda=0$, the fixed-time synchronization errors between the systems (65) and (67) are demonstrated in Figures 10-13, where 8 classes of different initial values are chosen for equations (65) and (67) by $x_{1}^{k}(s)=-0.4, x_{2}^{k}(s)=0.6, y_{1}^{k}(s)=-0.25+0.3 k$, and $y_{2}^{k}(s)=0.4-0.3 k$, for $s \in[-1,0]$ and $k \in\{-4$, 


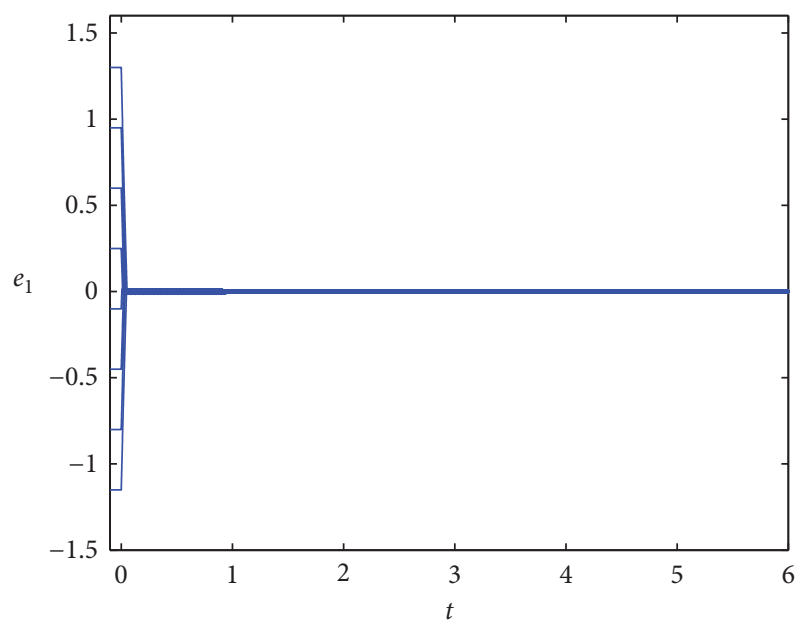

FIgURE 6: The fixed-time synchronization errors $e_{1}$ curves under controller (26), $\lambda>0$, in Example 1.

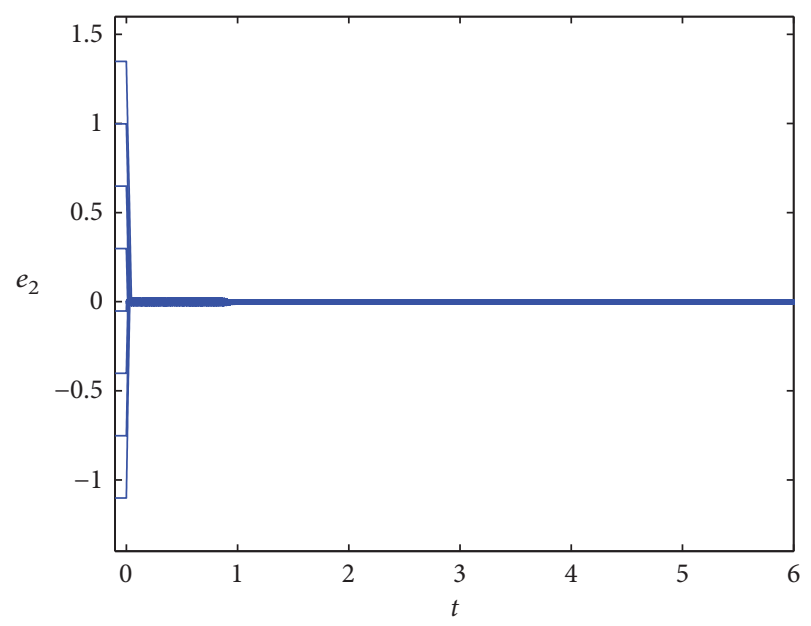

FIgURE 7: The fixed-time synchronization errors $e_{2}$ curves under controller (26), $\lambda>0$, in Example 1.

$-3,-2,-1,0,1,3\}$. Figures $10-13$ show that our results are effective, when $\lambda=0$. Through calculation, the settling time is estimated by $T \leq 2.049$.

Case (III): $-0.2=\lambda<0$.

When $\bar{\alpha}_{1}=3.545,-0.2=\alpha_{1}=-\bar{\alpha}_{1}-\underline{a}_{1}+(1 / 2) \sum_{j=1}^{2}$ $\bar{b}_{1 j} L_{j}+(1 / 2) \sum_{j=1}^{2} \bar{b}_{j 1} L_{1}<0$; when $\bar{\alpha}_{2}=3.809,-0.1=$ $\alpha_{2}=-\bar{\alpha}_{2}-\underline{a}_{2}+(1 / 2) \sum_{j=1}^{2} \bar{b}_{2 j} L_{j}+(1 / 2) \sum_{j=1}^{2} \bar{b}_{j 2} L_{2}<$ 0 , which implies that all the conditions of Theorem 1 are satisfied, and the drive-response systems (1) and (8) reach fixed-time synchronization.

When $\lambda<0$, the fixed-time synchronization errors between the systems (65) and (67) are demonstrated in Figures 14-17, where 8 classes of different initial values are chosen for equations (65) and (67) by $x_{1}^{k}(s)=-0.4, x_{2}^{k}(s)=0.6, y_{1}^{k}(s)=-0.3+0.35 k$, and

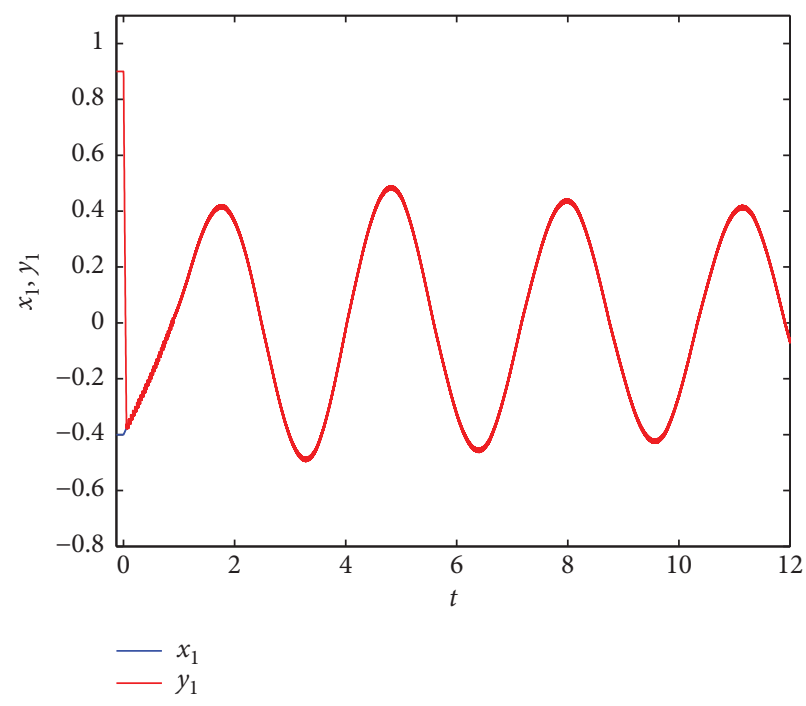

FIgURE 8: The fixed-time synchronization curves $x_{1}(t)$ and $y_{1}(t)$ under controller (26), $\lambda>0$, in Example 1.

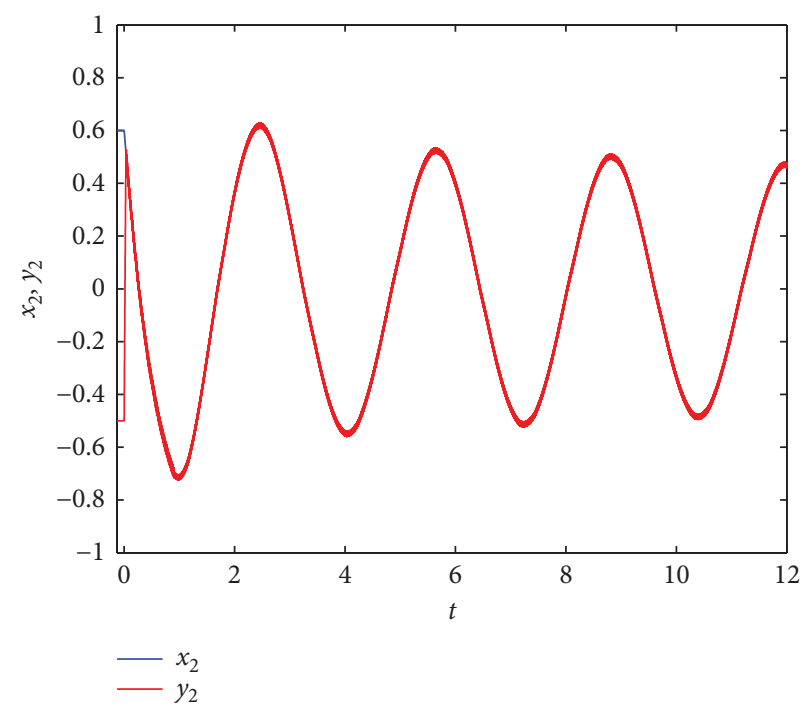

FIgURE 9: The fixed-time synchronization curves $x_{2}(t)$ and $y_{2}(t)$ under controller (26), $\lambda>0$, in Example 1.

$y_{2}^{k}(s)=0.5-0.4 k, \quad$ for $\quad s \in[-1,0] \quad$ and $k \in\{-4,-3,-2,-1,0,1,3\}$. Figures $14-17$ show that our results are effective, if $\lambda<0$. Through calculation, the settling time is estimated by $T \leq 1.91$.

Figures 6-17 demonstrate the correctness and effectiveness of the conclusions of Theorem 1.

Case (B): under controller (41).

Take the same controller parameters as in controller (26). By substituting the parameters of controller (26) into controller (41), we can derive 


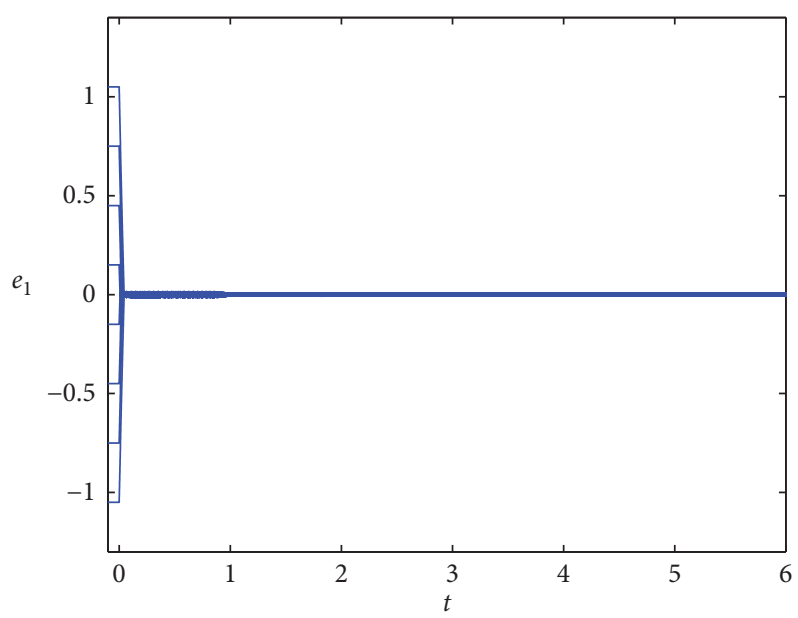

FIgURE 10: The fixed-time synchronization errors $e_{1}$ curves under controller $(26), \lambda=0$, in Example 1.

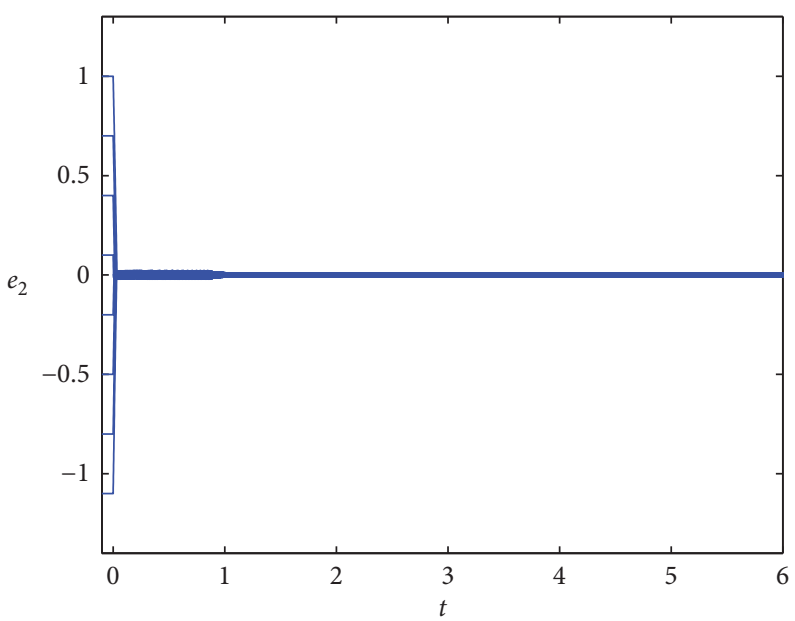

FIgURE 11: The fixed-time synchronization errors $e_{2}$ curves under controller (26), $\lambda=0$, in Example 1.

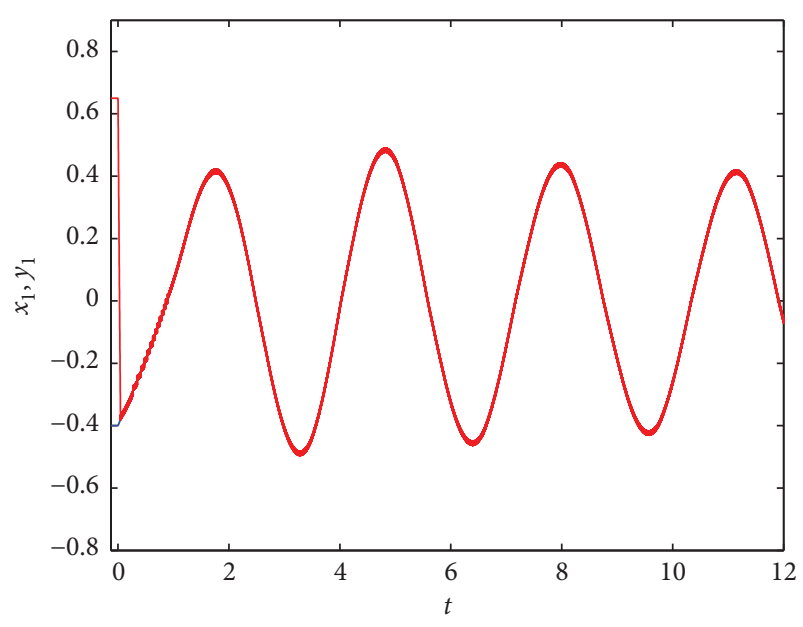

$-x_{1}$

FIGURE 12: The fixed-time synchronization curves $x_{1}(t)$ and $y_{1}(t)$ under controller (26), $\lambda=0$, in Example 1.

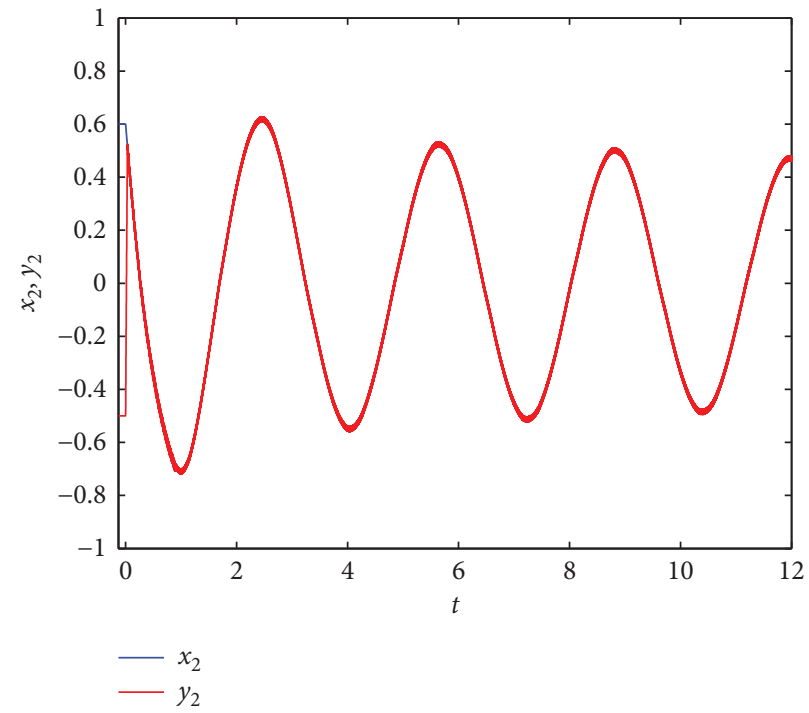

Figure 13: The fixed-time synchronization curves $x_{2}(t)$ and $y_{2}(t)$ under controller (26), $\lambda=0$, in Example 1. 


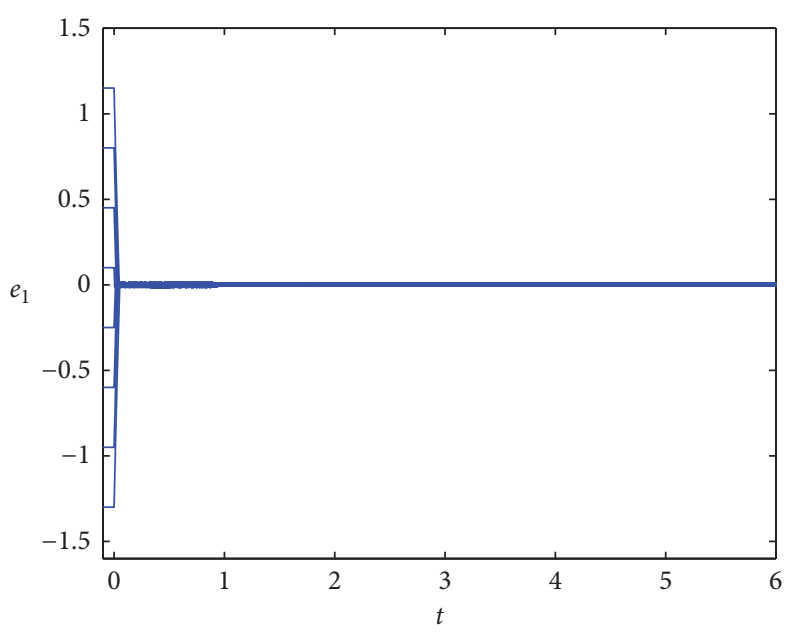

FIgURE 14: The fixed-time synchronization errors $e_{1}$ curves under controller $(26), \lambda<0$, in Example 1.

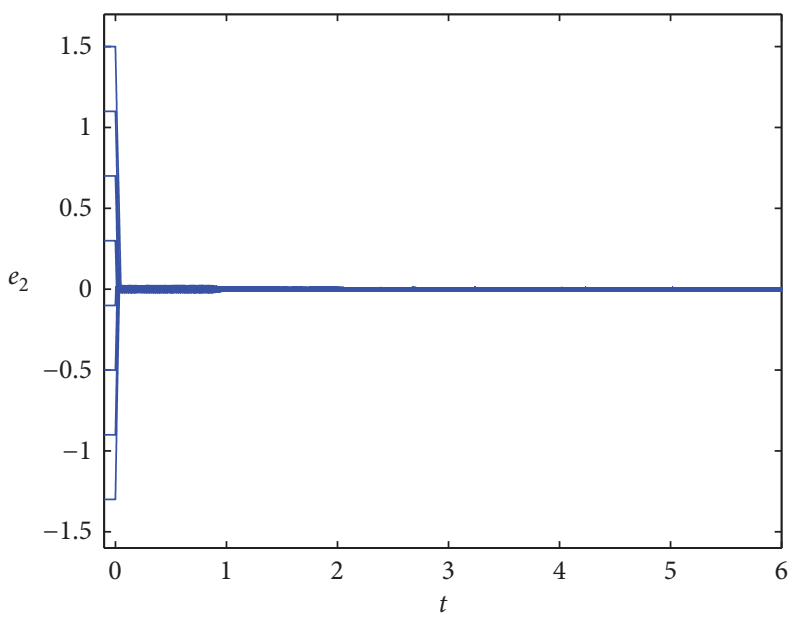

FIgURE 15: The fixed-time synchronization errors $e_{2}$ curves under controller $(26), \lambda<0$, in Example 1.

$$
\begin{aligned}
& K_{1}(t)=-\bar{\alpha}_{1 k} e_{1}(t)-\tanh \left(e_{1}(t)\right)\left(17+0.9\left|e_{1}(t)\right|^{3.1+2 \operatorname{sign}(V(t)-1)-1}-1.21\left|e_{1}\left(t-\tau_{1}(t)\right)\right|-1.595\left|e_{2}\left(t-\tau_{2}(t)\right)\right|\right) \\
& K_{2}(t)=-\bar{\alpha}_{2 k} e_{2}(t)-\tanh \left(e_{2}(t)\right)\left(23+0.9\left|e_{2}(t)\right|^{3.1+2 \operatorname{sign}(V(t)-1)-1}-1.76\left|e_{1}\left(t-\tau_{1}(t)\right)\right|-1.87\left|e_{2}\left(t-\tau_{2}(t)\right)\right|\right)
\end{aligned}
$$

where $k=1,2,3$. According to the control parameters $\bar{\alpha}_{1 k}$ and $\bar{\alpha}_{2 k}$, the numerical simulation is carried out in three cases, and the initial values are the same as those of Case (A), respectively.

It can be seen from Figures 18-20 that the control synchronization settling time $T$ of the drive-response systems (65) and (67) is the same as that of Case (A), respectively. Furthermore, by comparing Figures $18-20$ and $6-17$, it can be found that there is no chattering for the state trajectories of error systems under controller (41), in Example 1.
According to Figures 18-20, the conclusions of Corollary 1 are correct and effective.

4.2. Example of Fixed-Time Synchronization under Switching State-Feedback Controller

Example 2. Consider the 2-dimensional memristive neural networks with discontinuous neuron activation functions and mixed time-varying delays as follows: 


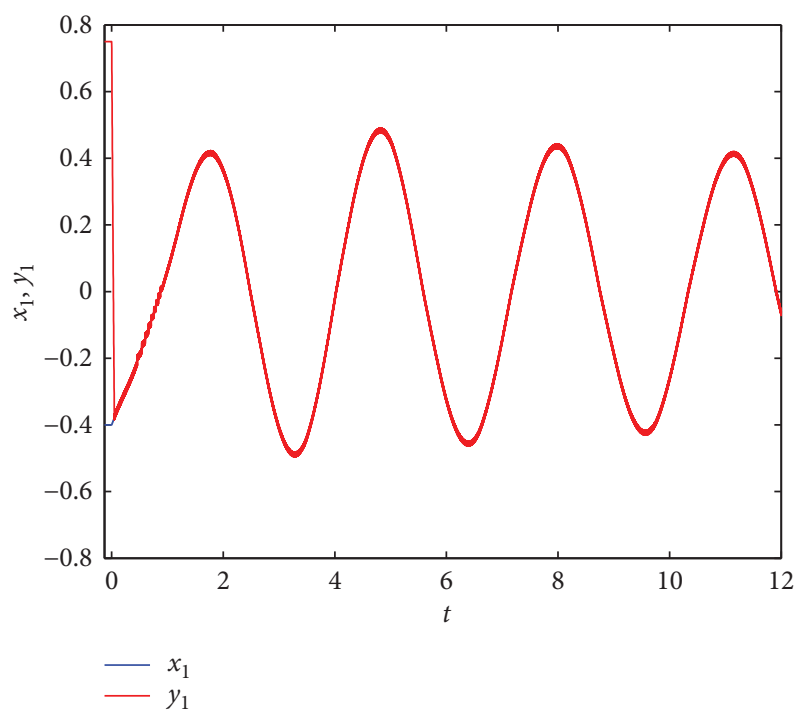

FIgURE 16: The fixed-time synchronization curves $x_{1}(t)$ and $y_{1}(t)$ under controller $(26), \lambda<0$, in Example 1.

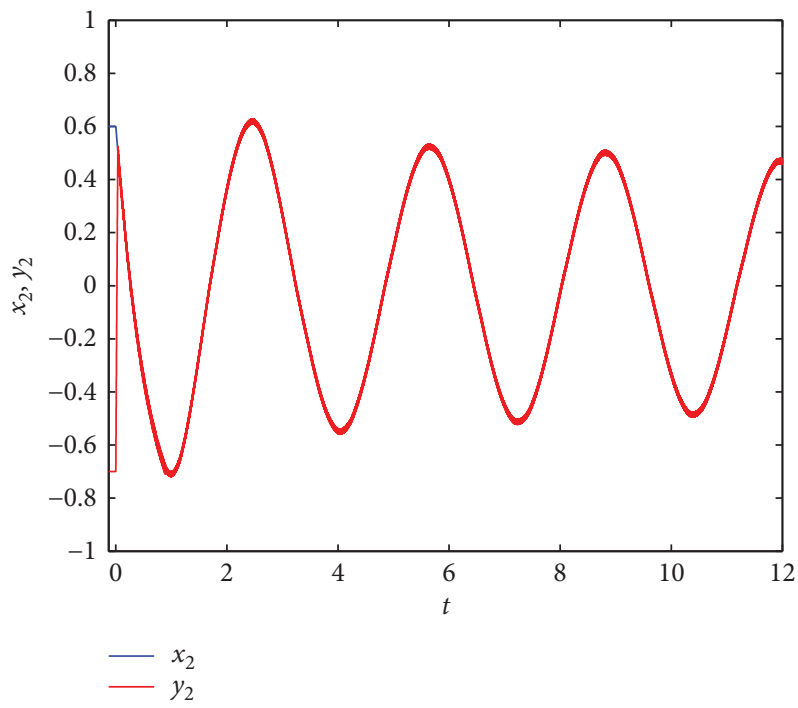

FIgURE 17: The fixed-time synchronization curves $x_{2}(t)$ and $y_{2}(t)$ under controller $(26), \lambda<0$, in Example 1 .

$$
\begin{aligned}
\dot{x}_{i}(t)= & -a_{i}\left(x_{i}(t)\right) x_{i}(t)+\sum_{j=1}^{2} b_{i j}\left(x_{j}(t)\right) f_{j}\left(x_{j}(t)\right)+\sum_{j=1}^{2} c_{i j}\left(x_{j}\left(t-\tau_{j}(t)\right)\right) \\
& \times g_{j}\left(x_{j}\left(t-\tau_{j}(t)\right)\right)+\sum_{j=1}^{2} d_{i j}\left(x_{j}(t)\right) \int_{t-\delta_{j}(t)}^{t} f_{j}\left(x_{j}(s)\right) \mathrm{d} s+I_{i},
\end{aligned}
$$

where $\left.\quad i=1,2, \quad f_{1}(u)\right)=f_{2}(u)=1.3 \tanh (u)-0.01 \operatorname{sign}$ $(u), g_{1}(u)=g_{2}(u)=\sin (u)-0.01 \operatorname{sign}(u) \tau_{1}(t)=\tau_{2}(t)=$ $\left(e^{t} / 1+e^{t}\right), \delta_{1}(t)=\delta_{2}(t)=1$, and $I_{1}=I_{2}=0$; and the values of the memristors are as follows: 


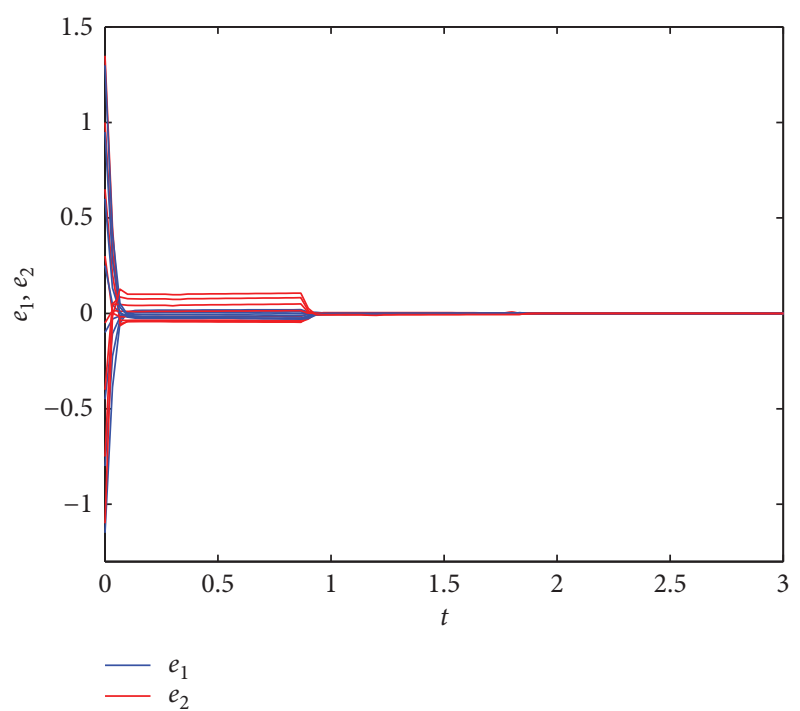

FIGURE 18: When $\lambda=0.4, \bar{\alpha}_{11}=3.245, \bar{\alpha}_{21}=3.509$, state trajectories of error systems $e_{1}$ and $e_{2}$ under controller (41), and the settling time $T \leq 2.4707$.

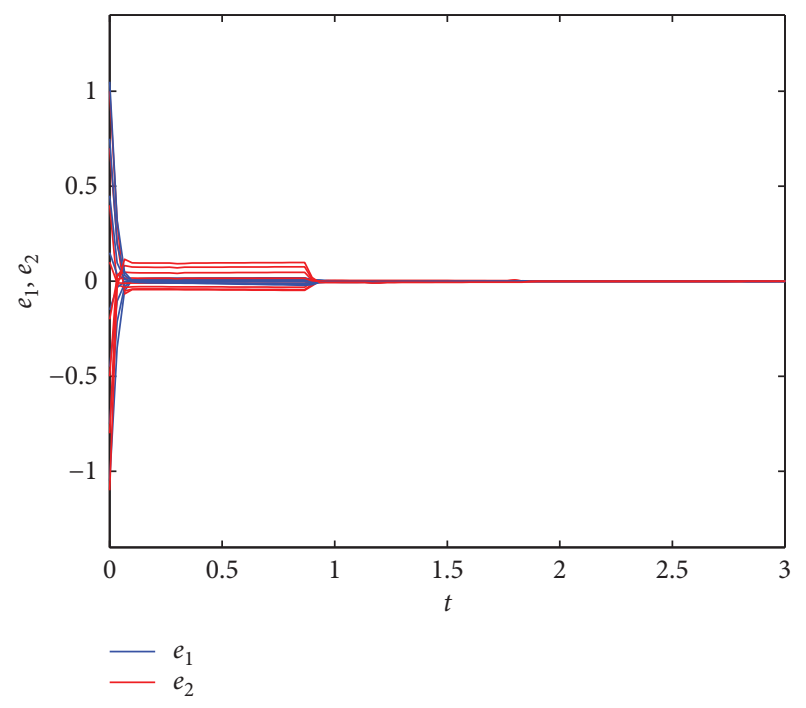

FIGURE 19: When $\lambda=0, \bar{\alpha}_{12}=3.245, \bar{\alpha}_{22}=3.709$, state trajectories of error systems $e_{1}$ and $e_{2}$ under controller (41), and the settling time $T \leq 2.049$.

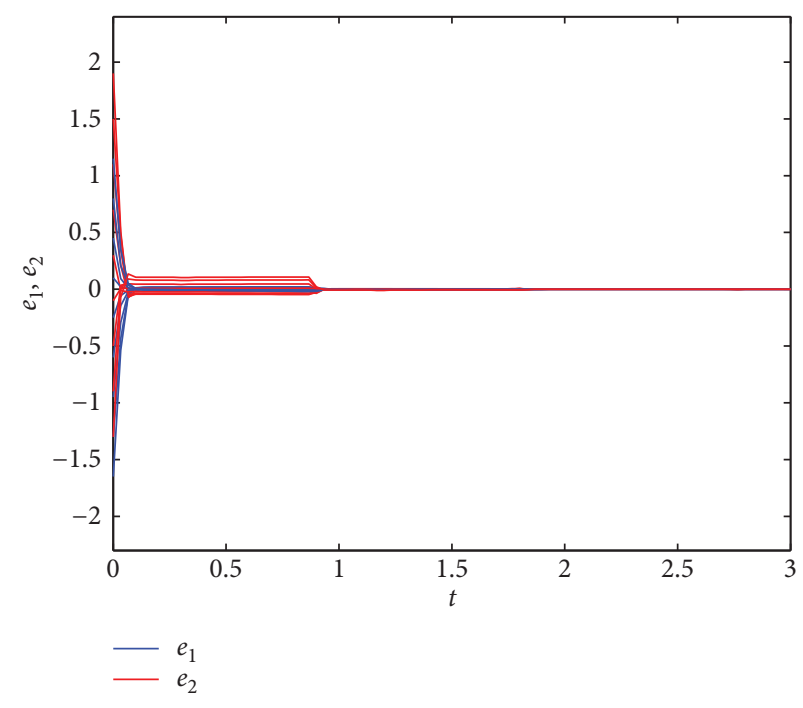

FIgURE 20: When $\lambda=-0.2, \bar{\alpha}_{13}=3.54, \bar{\alpha}_{23}=3.809$, state trajectories of error systems $e_{1}$ and $e_{2}$ under controller (41), and the settling time $T \leq 1.91$.

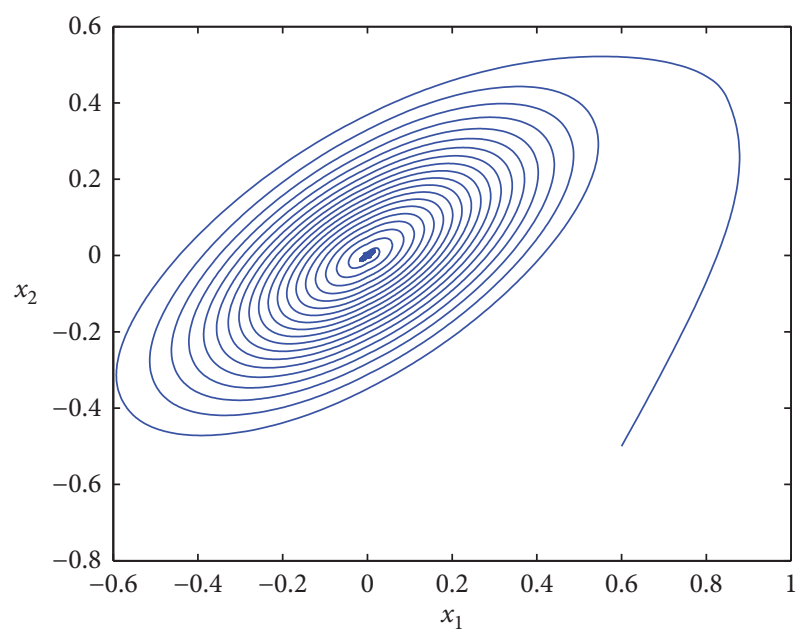

FIgURE 21: The chaotic attractors of system (69) with the initial values $x_{1}(s)=0.6$ and $x_{2}(s)=-0.5$, for $s \in[-1,0]$. 


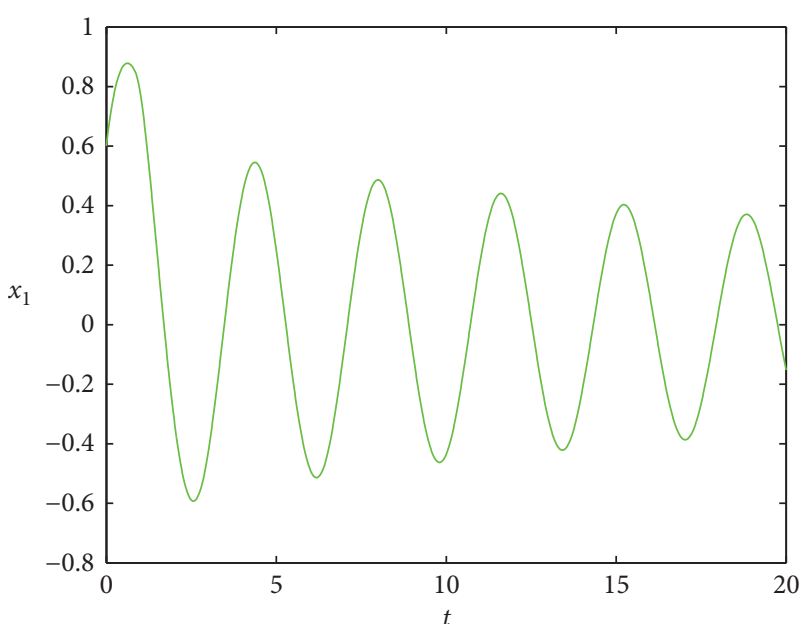

Figure 22: The dynamical behaviors of $x_{1}(t)$ of system (69) with the initial value $x_{1}(s)=0.6$, for $s \in[-1,0]$.

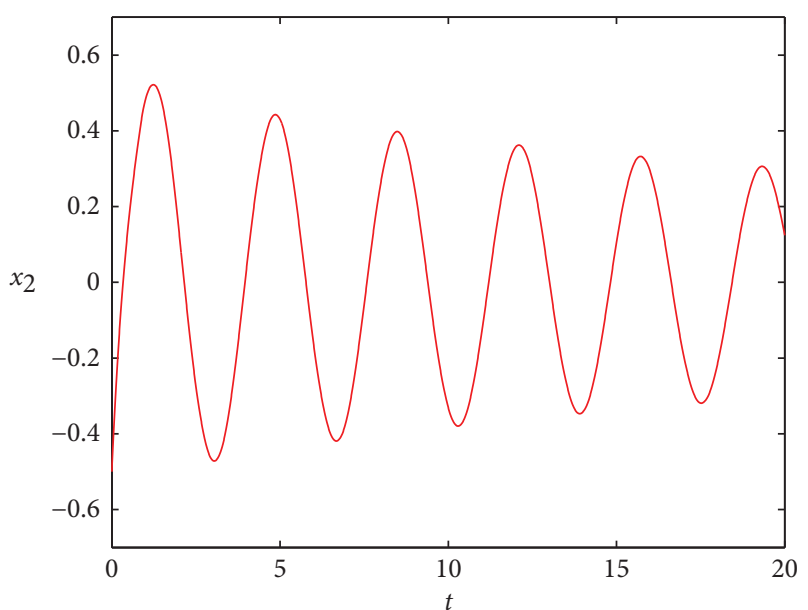

Figure 23: The dynamical behaviors of $x_{2}(t)$ of system (69) with the initial value $x_{2}(s)=-0.5$, for $s \in[-1,0]$.

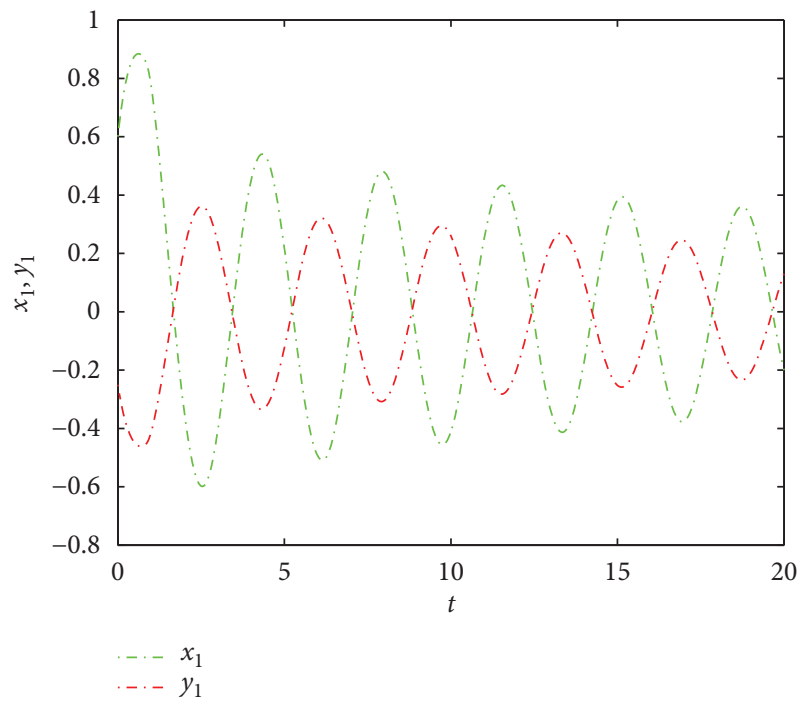

Figure 24: The state curves of $x_{1}(t)$ and $y_{1}(t)$ without control, in Example 2.

$$
\begin{aligned}
& a_{1}\left(x_{1}(t)\right)= \begin{cases}0.63, & \left|x_{1}(t)\right| \leq 1.2, \\
0.9, & \left|x_{1}(t)\right|>1.2,\end{cases} \\
& a_{2}\left(x_{2}(t)\right)= \begin{cases}-0.5, & \left|x_{2}(t)\right| \leq 1.2, \\
0.4, & \left|x_{2}(t)\right|>1.2,\end{cases} \\
& b_{11}\left(x_{1}(t)\right)= \begin{cases}-0.6, & \left|x_{1}(t)\right| \leq 1.2, \\
-0.5, & \left|x_{1}(t)\right|>1.2,\end{cases} \\
& b_{12}\left(x_{1}(t)\right)= \begin{cases}0.9, & \left|x_{1}(t)\right| \leq 1.2, \\
-1.3, & \left|x_{1}(t)\right|>1.2,\end{cases} \\
& d_{21}\left(x_{2}(t)\right)= \begin{cases}-1.23, & \left|x_{2}(t)\right| \leq 1.2, \\
-0.8, & \left|x_{2}(t)\right|>1.2,\end{cases} \\
& d_{21}\left(x_{2}(t)\right)= \begin{cases}1.2, & \left|x_{2}(t)\right| \leq 1.2, \\
-0.46, & \left|x_{2}(t)\right|>1.2,\end{cases} \\
& d_{22}\left(x_{2}(t)\right)= \begin{cases}-1,2, & \left|x_{2}(t)\right| \leq 1.2, \\
-1.1, & \left|x_{2}(t)\right| \leq 1.2,\end{cases} \\
& c_{22}\left(x_{2}(t)\right)= \begin{cases}1.1 .2, & \left|x_{2}(t)\right|>1.2, \\
0.9, & \left.\mid x_{1}(t)\right)\end{cases} \\
& c_{11}\left(x_{1}(t)\right)= \begin{cases}-0.35, & \left|x_{1}(t)\right| \leq 1.2, \\
-1.54, & \left|x_{1}(t)\right|>1.2,\end{cases}
\end{aligned}
$$

The model (69) has chaotic attractor with the initial values $x_{1}(s)=0.6$ and $x_{2}(s)=-0.5$ for $s \in[-1,0]$ which can be seen in Figure 21; and the state trajectories of system (69) with initial conditions $x_{1}(s)=0.6, x_{2}=-0.5$ are described in Figures 22 and 23.

Considering equation (69) as the drive system, the corresponding response system is described as follows: 


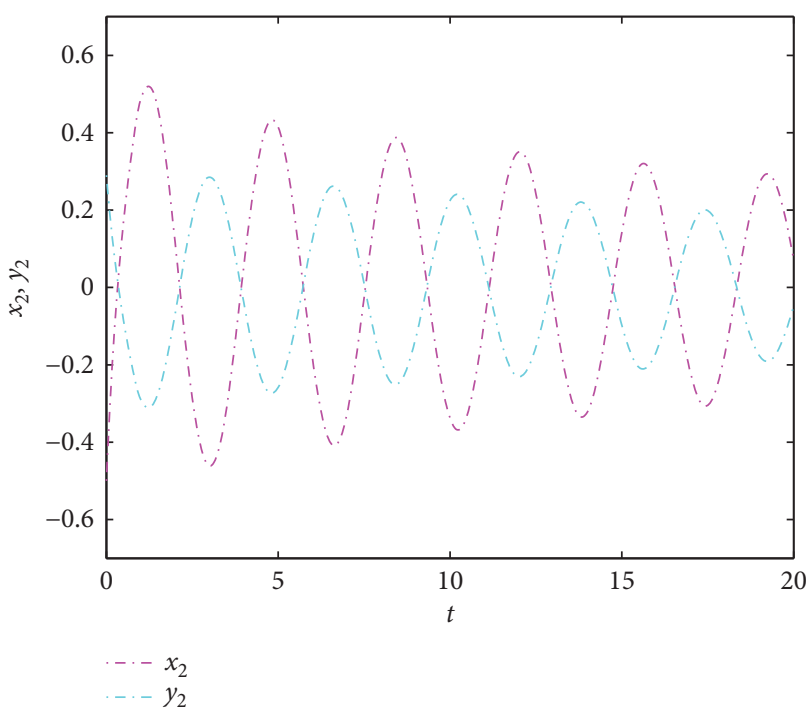

FIGURE 25: The state curves of $x_{2}(t)$ and $y_{2}(t)$ without control, in Example 2.

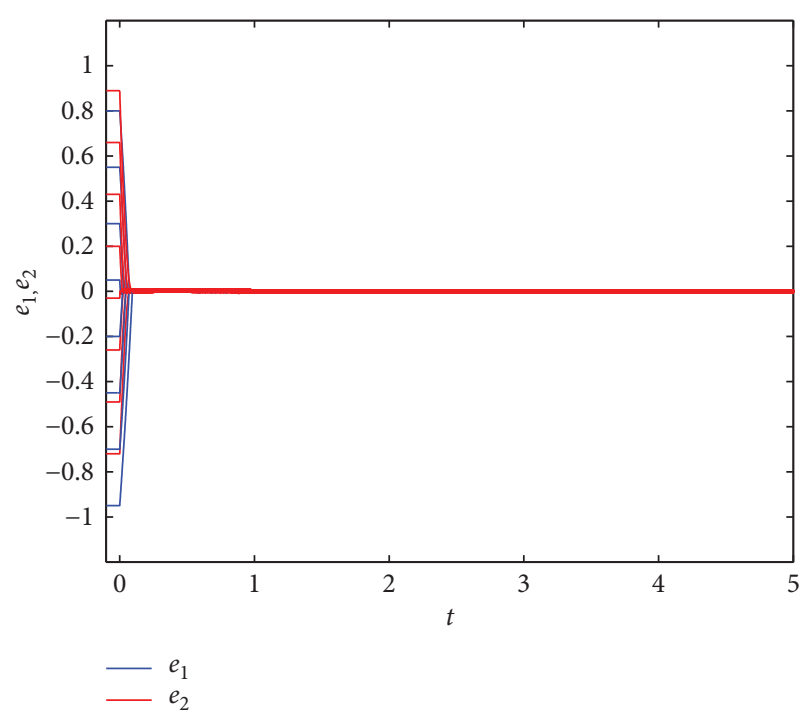

Figure 26: The fixed-time synchronization errors curves of $e_{1}$ and $e_{2}$ under controller (45), $\omega<0$, in Example 2.

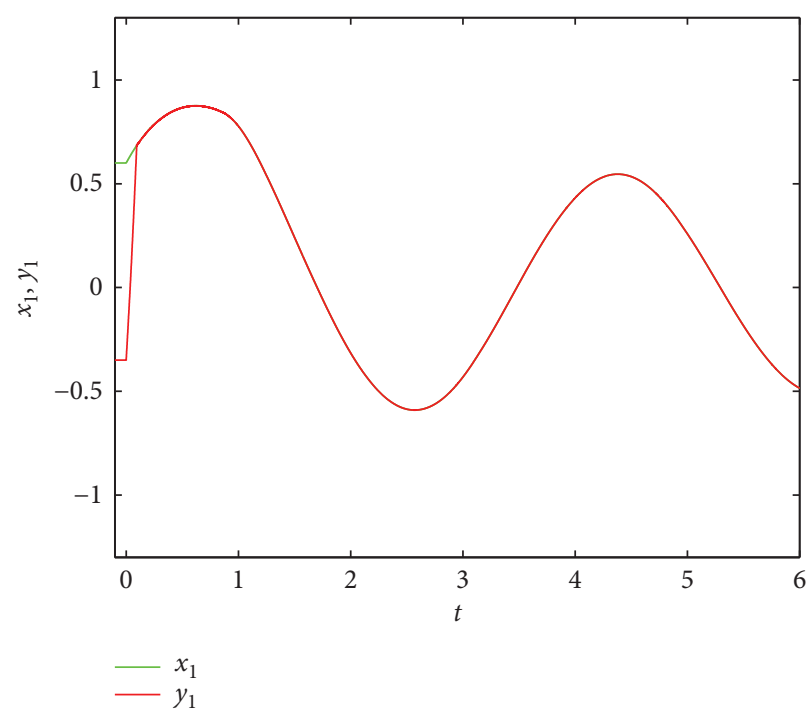

FIgURE 27: The fixed-time synchronization curves of $x_{1}(t)$ and $y_{1}(t)$ under controller (45), $\omega<0$, in Example 2.

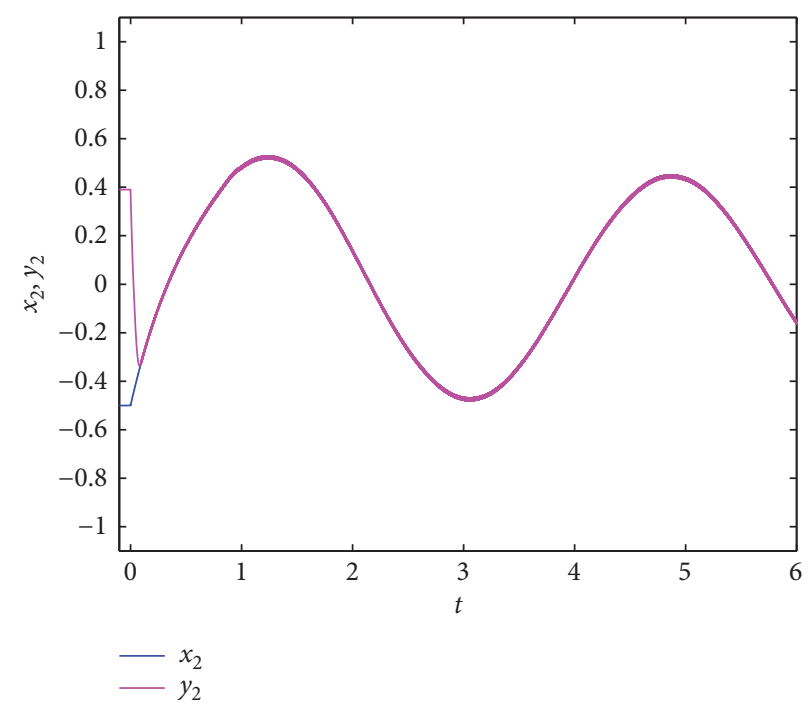

FIgURE 28: The fixed-time synchronization curves of $x_{2}(t)$ and $y_{2}(t)$ under controller (45), $\omega<0$, in Example 2.

$$
\begin{aligned}
\dot{y}_{i}(t)= & -a_{i}\left(y_{i}(t)\right) y_{i}(t)+\sum_{j=1}^{2} b_{i j}\left(y_{j}(t)\right) f_{j}\left(y_{j}(t)\right)+\sum_{j=1}^{2} c_{i j}\left(y_{j}\left(t-\tau_{j}(t)\right)\right) \\
& \times g_{j}\left(y_{j}\left(t-\tau_{j}(t)\right)\right)+\sum_{j=1}^{2} d_{i j}\left(y_{j}(t)\right) \int_{t-\delta_{j}(t)}^{t} f_{j}\left(y_{j}(s)\right) \mathrm{d} s+I_{i}+\bar{U}_{i}(t),
\end{aligned}
$$

where the parameters $a_{i}(\cdot), b_{i j}(\cdot), c_{i j}(\cdot), d_{i j}(\cdot), f_{j}(\cdot), \tau_{j}(\cdot)$, and $\delta_{j}(t)$ are the same as those defined in system (69).

Through calculation, we know that $L_{1}=L_{2}=1.3$, $N_{1}=N_{2}=0.01, \quad Z_{1}=Z_{2}=1, \quad H_{1}=H_{2}=0.01$, $S_{1}=S_{2}=1.29$, and $M_{1}=M_{2}=1.01$.

It is easy to check that the activation functions $f_{1}, f_{2}, g_{1}$, and $g_{2}$ satisfy assumptions $\left(H_{1}\right)-\left(H_{4}\right)$.
It is easy to get that $\underline{a}_{1}=0.63, \underline{a}_{2}=-0.5$, $\bar{a}_{1}=0.9, \bar{a}_{2}=0.4, \quad \bar{b}_{11}=0.6, \bar{b}_{12}=1.3, \bar{b}_{21}=1.23, \bar{b}_{22}=1.7$, $\bar{c}_{11}=1.54, \bar{c}_{12}=0.85, \bar{c}_{21}=1.3, \bar{c}_{22}=1.6$, and $\bar{d}_{11}=0.94$, $\bar{d}_{12}=1.15, \bar{d}_{21}=1.2, \bar{d}_{22}=1.2$.

Take the control parameters $q_{1}=15.8$ and $q_{2}=21$. By simple calculations, $0.1932=q_{1}-\left|a_{1}^{*}-a_{1}^{* *}\right| T_{1}-\sum_{j=1}^{2} \bar{b}_{j 1}$ $\left(N_{j}+2 S_{j}\right)-\sum_{j=1}^{2} \bar{c}_{1 j}\left(H_{j}+2 M_{j}\right)-2 \sum_{j=1}^{2} \bar{d}_{1 j} S_{j} \delta_{j} \geq 0$, and 


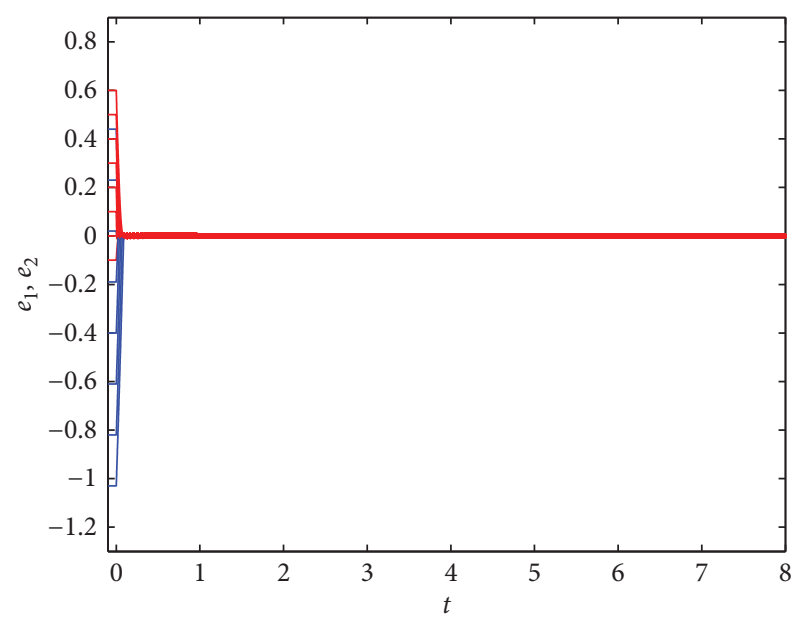

$$
-e_{1}
$$

FIgURE 29: The fixed-time synchronization errors curves of $e_{1}$ and $e_{2}$ under controller (45), $\omega>0$, in Example 2.

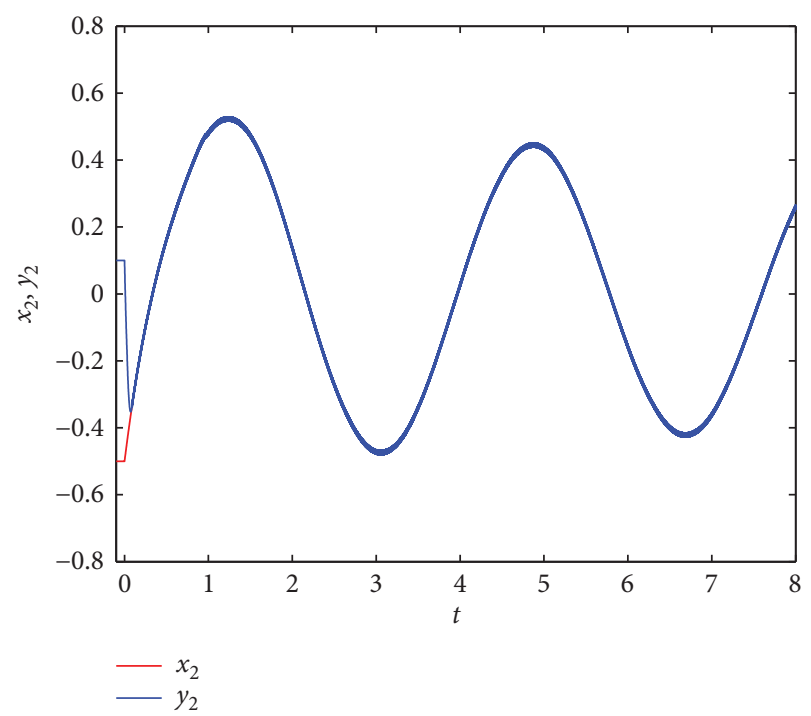

Figure 30: The fixed-time synchronization curves of $x_{1}(t)$ and $y_{1}(t)$ under controller (45), $\omega>0$, in Example 2.

$0.2576=q_{2}-\quad\left|a_{2}^{*}-a_{2}^{* *}\right| T_{2}-\sum_{j=1}^{2} \bar{b}_{j 2}\left(N_{j}+2 S_{j}\right)-\sum_{j=1}^{2} \bar{c}_{2 j}$
$\left(H_{j}+2 M_{j}\right)-2 \sum_{j=1}^{2} \bar{d}_{2 j} S_{j} \delta_{j} \geq 0$.

It can be seen from Figures 24 and 25 that when no controller is added to the response system (71), the state curves do not achieve fixed-time synchronization, in Example 2 .

We take the control parameters $\curlywedge=0.9$ and $\gamma=1.5$. Next, we will calculate parameter $\omega$ about the control gain $\bar{\omega}_{i}$ in three cases:

Case (I): $-0.1=\omega<0$.

When $\bar{\omega}_{1}=1.849,-0.1=\omega_{1}=-\bar{\omega}_{1}-\underline{a}_{1}+\sum_{j=1}^{2} \bar{b}_{1 j} L_{j}$ $<0$; when $\bar{\omega}_{2}=4.5,-0.1=\omega_{2}=-\bar{\omega}_{2}-\underline{a}_{2}+\sum_{j=1}^{2} \bar{b}_{2 j}$ $L_{j}<0$; it can be found that all the conditions of

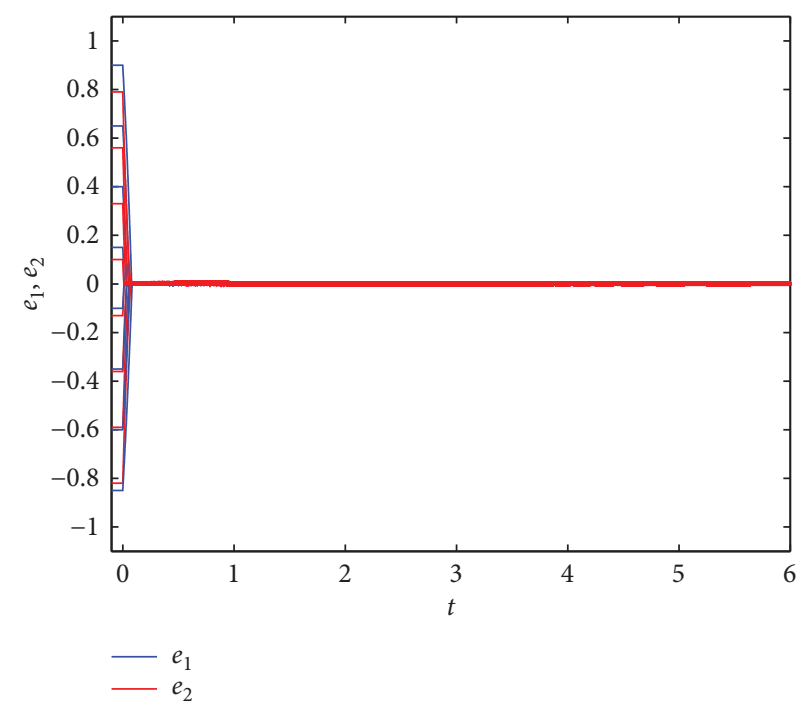

FIgURE 31: The fixed-time synchronization curves of $x_{2}(t)$ and $y_{2}(t)$ under controller (45), $\omega>0$, in Example 2.

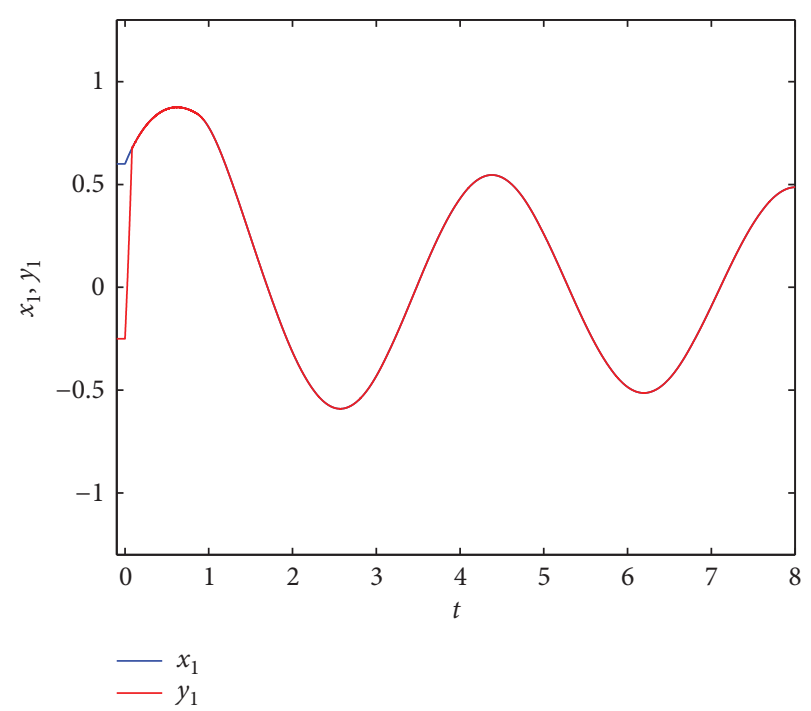

FIgURE 32: The fixed-time synchronization errors curves of $e_{1}$ and $e_{2}$ under controller (45), $\omega=0$, in Example 2.

Theorem 2 are satisfied, and the drive-response systems (1) and (8) achieve fixed-time synchronization.

When $\omega<0$, the fixed-time synchronization errors between the system (69) and (71) are demonstrated in Figures 26-28, where 8 classes of different initial values are chosen for (69) and (71) by $x_{1}^{k}(s)=0.6$, $x_{2}^{k}(s)=-0.5, y_{1}^{k}(s)=0.4-0.25 k$, and $y_{2}^{k}(s)=-0.3+$ $0.23 k$, for $s \in[-1,0]$ and $k \in\{-4,-3,-2,-1,0,1,3\}$. Figures 26-28 show that our results are effective when $\omega<0$. Through calculation, the settling time is estimated to be $T \leq 3.9291$. We can see that our conclusion of Theorem 2 is correct.

Case (II): $0.1=\omega>0$. 


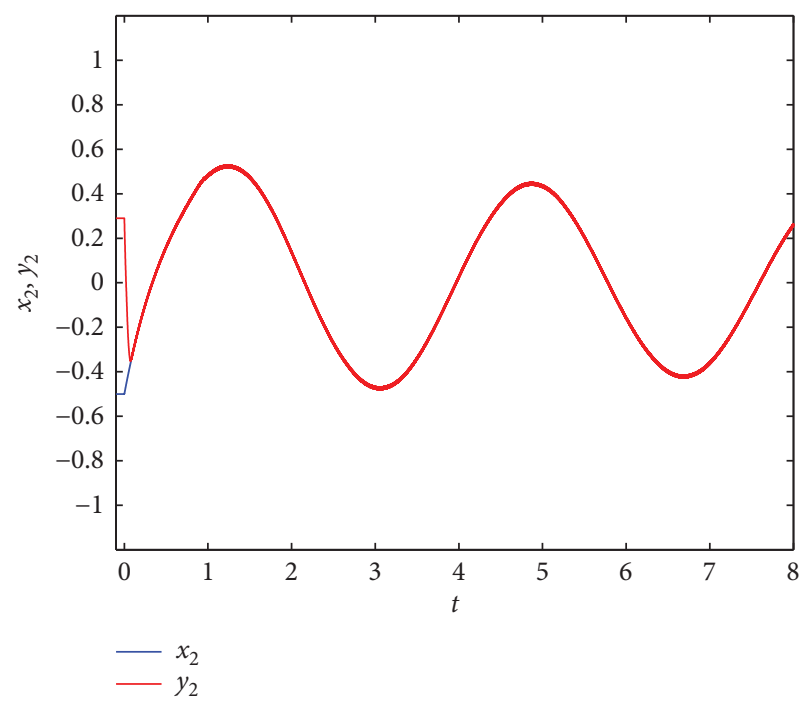

FIGURE 33: The fixed-time synchronization errors curves of $x_{1}(t)$ and $y_{1}(t)$ under controller (45), $\omega=0$, in Example 2.

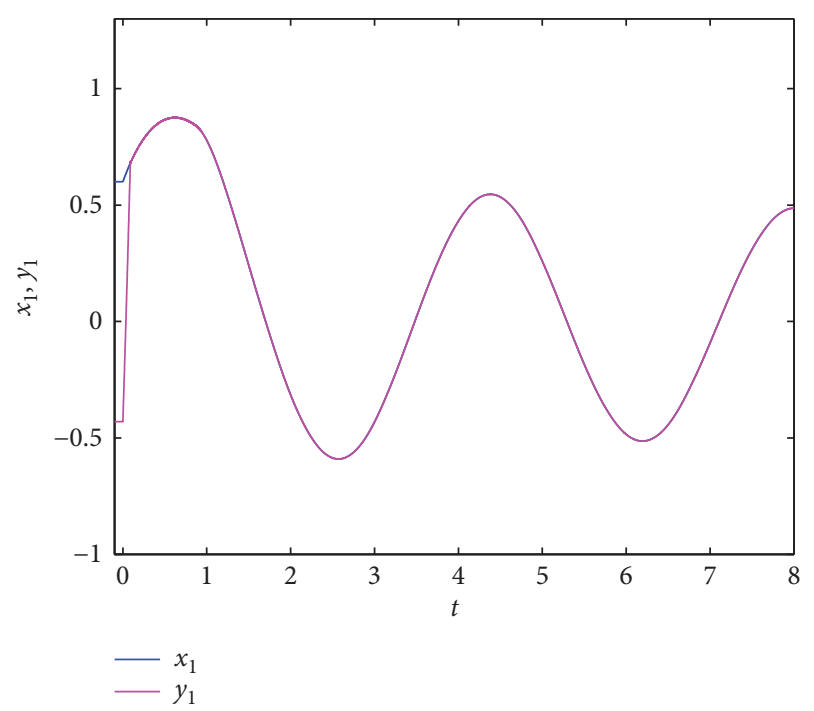

FIgURE 34: The fixed-time synchronization errors curves of $x_{2}(t)$ and $y_{2}(t)$ under controller (45), $\omega=0$, in Example 2.

When $\quad \bar{\omega}_{1}=1.649, \quad 0.1=\omega_{1}=-\bar{\omega}_{1}-\underline{a}_{1}+\sum_{j=1}^{2} \bar{b}_{1 j} L_{j}$ $\geq 0 ; \quad$ when $\quad \bar{\omega}_{2}=4.3, \quad 0.1=\omega_{2}=-\bar{\omega}_{2}-\underline{a}_{2}+\sum_{j=1}^{2}$ $\bar{b}_{2 j} L_{j}>0$; it can be found that all the conditions of Theorem 2 are satisfied, and the drive-response systems (1) and (8) achieve fixed-time synchronization.

When $\omega>0$, the fixed-time synchronization errors between the system (69) and (71) are demonstrated in Figures 29-31, where 8 classes of different initial values are chosen for equations (69) and (71) by $x_{1}^{k}(s)=0.6$, $x_{2}^{k}(s)=-0.5, y_{1}^{k}(s)=0.2-0.21 k$, and $y_{2}^{k}(s)=-0.2+$ $0.1 k$, for $s \in[-1,0]$ and $k \in\{-4,-3,-2,-1,0,1,3\}$. Figures 29-31 show that our results are effective when $\omega>0$. Through calculation, the settling time is estimated by $T \leq 5.0061$. We can know that our conclusion of Theorem 2 is correct.
Case (III): $0=\omega$.

When $\quad \bar{\omega}_{1}=1.749, \quad 0=\omega_{1}=-\bar{\omega}_{1}-\underline{a}_{1}+\sum_{j=1}^{2} \bar{b}_{1 j} L_{j}$; when $\bar{\omega}_{2}=4.4,0=\omega_{2}=-\bar{\omega}_{2}-\underline{a}_{2}+\sum_{j=1}^{2} \bar{b}_{2 j} L_{j}$, which implies that all the conditions of Theorem 2 are satisfied, and the drive-response systems (1) and (8) achieve fixed-time synchronization.

When $\omega=0$, the fixed-time synchronization errors between the systems (69) and (71) are demonstrated in Figures 32-34, where 8 classes of different initial values are chosen for equations (69) and (71) by $x_{1}^{k}(s)=0.6$, $x_{2}^{k}(s)=-0.5, y_{1}^{k}(s)=0.5-0.21 k$, and $y_{2}^{k}(s)=-0.4+$ $0.23 k$, for $s \in[-1,0]$ and $k \in\{-4,-3,-2,-1,0,1,3\}$. Figures 32-34 show that our results are effective, when $\omega>0$. Through calculation, the settling time is estimated by $T \leq 4.3147$. We can know that our conclusion of Theorem 2 is correct.

From Figures 26-34, it is clear that the conclusions of Theorem 2 are correct and effective.

\section{Conclusions}

In this paper, two energy-saving and simple controllers are designed to achieve the fixed-time synchronization for a class of memristive neural networks with discontinuous neuron activation functions and mixed time-varying delays. Under the fixed-time stability theory, the extended Filippov framework, and the theory of differential inclusion, several new and useful sufficient conditions of fixedtime synchronization for the drive-response systems (1) and (8) are obtained; and the power exponent in the controllers is a function rather than a constant, which can save energy. Meanwhile, the control parameters are closely related not only to the distributed delays but also to the switching jumps of state and have an inhibition effect on the fixed-time synchronization. In our model, the discontinuous activation function and mixed time-varying delays are taken into consideration, making our model more practical and realistic. It is verified by numerical simulations that the chattering phenomenon can be weakened or eliminated by replacing discontinuous sign functions with continuous tanh in the controllers. In addition, based on the above methods, the settling time of fixed-time synchronization proposed in this paper is closer to the actual synchronization time, which has made some progress on the basis of previous works [41, 42, 44, 55]. Finally, two numerical simulations are provided to verify the correctness and effectiveness of the obtained theoretical results. Shortly, we will consider the fixed-time synchronization of delayed memristive neural networks, design more energy-saving and simpler aperiodic intermittent controllers, and eliminate the bounded conditions of neuron activation functions.

\section{Data Availability}

The datasets generated and/or analysed during the current study are available from the corresponding author upon reasonable request. 


\section{Conflicts of Interest}

The authors declare that they have no conflicts of interest.

\section{Acknowledgments}

This work was supported by the National Natural Science Foundation of People's Republic of China (Grant no. 12061055), Ningxia Natural Science Foundation (Grant nos. 2020AAC03030 and 2021AAC03175), Youth Science and Technology Talents Development Project of Guizhou Education Department (Qian Jiao he KY zi [2018] 313), Zunyi Normal College 2017 Academic New Talent Cultivation and Innovation Exploration Project (Qiankehe platform talent [2017], Grant no. 5727-24), and the First Class Discipline Construction Project in Ningxia Universities: Mathematics.

\section{References}

[1] L. Chua, "Memristor-the missing circuit element," IEEE Transactions on Circuit Theory, vol. 18, no. 5, pp. 507-519, 1971.

[2] D. B. Strukov, G. S. Snider, D. R. Stewart, and R. S. Williams, "The missing memristor found," Nature, vol. 453, no. 7191, pp. 80-83, 2008.

[3] S. P. Adhikari, C. Yang, H. Kim, and L. O. Chua, "Memristor bridge synapse-based neural network and its learning," IEEE Transactions on Neural Networks and Learning Systems, vol. 23, no. 9, pp. 1426-1435, 2012.

[4] S. P. Adhikari, H. Kim, B. Ram Kaji, C. Yang, and L. O. Chua, "A circuit-based learning architecture for multilayer neural networks with memristor bridge synapses," IEEE Transactions on Circuits and Systems I: Regular Papers, vol. 62, no. 1, pp. 215-223, 2015.

[5] Z. I. Mannan, S. P. Adhikari, C. Yang, B. Ram Kaji, H. Kim, and L. Chua, "Memristive imitation of synaptic transmission and plasticity," IEEE Transactions on Neural Networks and Learning Systems, vol. 30, no. 11, pp. 3458-3470, 2019.

[6] X. Shi, S. Duan, L. Wang, T. Huang, and C. Li, "A novel memristive electronic synapse-based hermite chaotic neural network with application in cryptography," Neurocomputing, vol. 166, pp. 487-495, 2015.

[7] F. Cai, S. Kumar, T. Van Vaerenbergh et al., "Power-efficient combinatorial optimization using intrinsic noise in memristor hopfield neural networks," Nature Electronics, vol. 3, no. 7, pp. 409-418, 2020.

[8] J. Sun, J. Han, P. Liu, and Y. Wang, "Memristor-based neural network circuit of pavlov associative memory with dual mode switching," AEÜ-International Journal of Electronics and Communications, vol. 129, Article ID 153552, 2021.

[9] C. Xiu, R. Zhou, and Y. Liu, "New chaotic memristive cellular neural network and its application in secure communication system," Chaos Solitons and Fractals, vol. 141, Article ID 110316, 2020.

[10] W. Wang, X. Yu, X. Luo, and L. Li, "Stability analysis of memristive multidirectional associative memory neural networks and applications in information storage," Modern Physics Letters B, vol. 32, no. 18, Article ID 1850207, 2018.

[11] X. Liu, C. Zhang, and L. Zhou, "Synchronization of coupled reaction-diffusion neural networks with hybrid coupling via aperiodically intermittent pinning control," Journal of the Franklin Institute, vol. 354, pp. 7053-7076, 2017.
[12] C. Hu, J. Yu, H. Jiang, and Z. Teng, "Exponential synchronization for reaction-diffusion networks with mixed delays in terms of p-norm via intermittent driving," Neural Networks, vol. 31, pp. 1-11, 2012.

[13] X. Song, J. Man, S. Song, and C. K. Ahn, "Finite/fixed-time anti-synchronization of inconsistent markovian quaternionvalued memristive neural networks with reaction-diffusion terms," IEEE Transactions on Circuits and Systems I-Regular Papers, vol. 68, no. 1, pp. 363-375, 2021.

[14] L. Duan, W. Hui, and L. Huang, "Finite-time synchronization of delayed fuzzy cellular neural networks with discontinuous activations," Fuzzy Sets and Systems, vol. 361, pp. 56-70, 2019.

[15] T. Yu, J. Cao, L. Rutkowski, and Y. Luo, "Finite-time synchronization of complex-valued memristive-based neural networks via hybrid control," IEEE Transactions on Neural Networks and Learning Systems, 2021.

[16] X. Wang, S. Kun, S. Zhong, and J. Cheng, "Exponential synchronization of memristor-based neural networks with time-varying delay and stochastic perturbation," Neurocomputing, vol. 242, pp. 131-139, 2017.

[17] J. Liu, S. Song, H. Jiang, J. Li, and X. Liu, "New results of projective synchronization for memristor-based coupled neural networks," Physica A: Statistical Mechanics and its Applications, vol. 545, Article ID 123739, 2020.

[18] L. Ning and W. X. Zheng, "Bipartite synchronization for inertia memristor-based neural networks on coopetition networks," Neural Networks, vol. 124, pp. 39-49, 2020.

[19] S. Shao, X. Liu, and J. Cao, "Prespecified-time synchronization of switched coupled neural networks via smooth controllers," Neural Networks, vol. 133, pp. 32-39, 2021.

[20] C. Zhou, C. Wang, Y. Sun, and W. Yao, "Weighted sum synchronization of memristive coupled neural networks," Neurocomputing, vol. 403, pp. 211-223, 2020.

[21] H. Meng, W. Chen, J. Zhang et al., "Finite-time synchronization of memristor-based fractional order Cohen-Grossberg neural networks," IEEE Access, vol. 8, pp. 73698-73713, 2020.

[22] C. Hu, J. Yu, Z. Chen, H. Jiang, and T. Huang, "Fixed-time stability of dynamical systems and fixed-time synchronization of coupled discontinuous neural networks," Neural Networks, vol. 89, pp. 74-83, 2017.

[23] Y. Wu, Y. Gao, and W. Li, "Finite-time synchronization of switched neural networks with state-dependent switching via intermittent control," Neurocomputing, vol. 384, pp. 325-334, 2020.

[24] A. Polyakov, "Nonlinear feedback design for fixed-time stabilization of linear control systems," IEEE Transactions on Automatic Control, vol. 57, no. 8, pp. 2106-2110, 2012.

[25] S. Dong, H. Zhu, S. Zhong, K. Shi, and Y. Liu, "New study on fixed-time synchronization control of delayed inertial memristive neural networks," Applied Mathematics and Computation, vol. 399, Article ID 126035, 2021.

[26] C. Chen, L. Li, H. Peng, Y. Yang, L. Mi, and B. Qiu, "Fixedtime projective synchronization of memristive neural networks with discrete delay," Physica A: Statistical Mechanics and its Applications, vol. 534, p. 122248, 2019.

[27] M. Zhang, L. Li, H. Peng, and J. Xiao, "Fixed-time synchronization of memristive fuzzy BAM cellular neural networks with time-varying delays based on feedback controllers,” IEEE Access, vol. 6, pp. 12085-12102, 2018.

[28] F. Ren, M. Jiang, H. Xu, and M. Li, "Quasi fixed-time synchronization of memristive Cohen-Grossberg neural networks with reaction-diffusion," Neurocomputing, vol. 415, pp. 74-83, 2020. 
[29] C. Yang, L. Huang, and Z. Cai, "Fixed-time synchronization of coupled memristor-based neural networks with time-varying delays," Neural Networks, vol. 116, pp. 101-109, 2019.

[30] M. P. Kennedy and L. O. Chua, "Neural networks for nonlinear programming," IEEE Transactions on Circuits and Systems, vol. 35, pp. 554-562, 1998.

[31] L. Li and L. Huang, "Global asymptotic stability of delayed neural networks with discontinuous neuron activations," Neurocomputing, vol. 72, pp. 3726-3733, 2009.

[32] L. Wang, Z. Zen, J. Hu, and X. Wang, "Controller design for global fixed-time synchronization of delayed neural networks with discontinuous activations," Neural Networks, vol. 87, pp. 122-131, 2017.

[33] Z. Cai, L. Huang, and L. Zhang, "New exponential synchronization criteria for time-varying delayed neural networks with discontinuous activations," Neural Networks, vol. 65, pp. 105-114, 2015.

[34] F. Kong, Q. Zhu, R. Sakthive, and A. Mohammadzadeh, "Fixed-time synchronization analysis for discontinuous fuzzy inertial neural networks with parameter uncertainties," Neurocomputing, vol. 422, pp. 295-313, 2021.

[35] L. Hui, W. He, Q. Han, and C. Peng, "Fixed-time synchronization for coupled delayed neural networks with discontinuous or continuous activations," Neurocomputing, vol. 314, pp. 143-153, 2018.

[36] L. Wang, Z. Zeng, X. Zong, and M. Ge, "Finite-time stabilization of memristor-based inertial neural networks with discontinuous activations and distributed delays," Journal of the Franklin Institute, vol. 356, pp. 3628-3643, 2019.

[37] L. Wang, H. He, and Z. Zeng, "Global synchronization of fuzzy memristive neural networks with discrete and distributed delays," IEEE Transactions on Fuzzy Systems, vol. 28, no. 9, pp. 2022-2034, 2020.

[38] W. Wang, X. Jia, X. Luo, J. Kurths, and M. Yuan, "Fixed-time synchronization control of memristive MAM neural networks with mixed delays and application in chaotic secure communication," Chaos Solitons and Fractals, vol. 126, pp. 85-96, 2019.

[39] Y. Feng, X. Yang, Q. Song, and J. Cao, "Synchronization of memristive neural networks with mixed delays via quantized intermittent control," Applied Mathematics and Computation, vol. 339, pp. 874-887, 2018.

[40] M. Yan and M. Jiang, "Synchronization with general decay rate for memristor-based BAM neural networks with distributed delays and discontinuous activation functions," Neurocomputing, vol. 387, pp. 221-240, 2020.

[41] A. Abdurahman, H. Jiang, and C. Hu, "General decay synchronization of memristor-based Cohen-Grossberg neural networks with mixed time-delays and discontinuous activations," Journal of the Franklin Institute, vol. 354, pp. 70287052, 2017.

[42] Y. Li, B. Luo, D. Liu, Z. Yang, and Y. Zhu, "Adaptive synchronization of memristor-based neural networks with discontinuous activations," Neurocomputing, vol. 381, pp. 196-206, 2020.

[43] A. Abdurahman and H. Jiang, "New results on exponential synchronization of memristor-based neural networks with discontinuous neuron activations," Neural Networks, vol. 84, pp. 161-171, 2016.

[44] X. Li, W. Zhang, J.-A. Fang, and H. Li, "Finite-time synchronization of memristive neural networks with discontinuous activation functions and mixed time-varying delays," Neurocomputing, vol. 340, pp. 99-109, 2019.
[45] J. Aubin and H. frankowska, Set-Valued Analysis, Birkhauser, Basel, Switzerland, 1990.

[46] N. Li, X. Wu, J. Feng, and J. Lu, "Fixed-time synchronization of complex dynamical networks: a novel and economical mechanism," IEEE Transactions on Cybernetics, vol. 19, pp. 1-11, 2020.

[47] L. Mi, C. Chen, B. Qiu, L. Xu, and L. Zhang, "Fixed-time synchronization analysis for complex-valued neural networks via a new fixed-time stability theorem," IEEE Access, vol. 8, pp. 172799-172807, 2020.

[48] Y. Zhang, J. Zhuang, Y. Xia, Y. Bai, J. Cao, and L. Gu, "Fixedtime synchronization of the impulsive memristor-based neural networks," Communications in Nonlinear Science and Numerical Simulation, vol. 77, pp. 40-53, 2019.

[49] F. Kong, Q. Zhu, and R. Sakthivel, "Finite-time and fixed-time synchronization control of fuzzy Cohen-Grossberg neural networks," Fuzzy Sets and Systems, vol. 394, pp. 87-109, 2020.

[50] C. Chen, L. Li, H. Peng, J. Kurths, and Y. Yang, "Fixed-time synchronization of hybrid coupled networks with timevarying delays," Chaos, Solitons \& Fractals, vol. 108, pp. 4956, 2018.

[51] A. Abdurahman, H. Jiang, and Z. Teng, "Finite-time synchronization for fuzzy cellular neural networks with timevarying delays," Fuzzy Sets and Systems, vol. 297, pp. 96-111, 2016.

[52] Z. Cai, L. Huang, and L. Zhang, "Improved switching controllers for finite-time synchronization of delayed neural networks with discontinuous activations," Journal of the Franklin Institute, vol. 354, pp. 6692-6723, 2017.

[53] J. Li, H. Jiang, C. Hu, and A. Ahmed, "Finite/fixed-time synchronization control of coupled memristive neural networks," Journal of the Franklin Institute, vol. 356, pp. 99289952, 2019.

[54] J. Gao, P. Zhu, A. Ahmed, F. E. Alsaadi, and T. Hayat, “A new switching control for finite-time synchronization of memristor-based recurrent neural networks," Neural Networks, vol. 86, pp. 1-9, 2017.

[55] C. Chen, L. Li, H. Peng, Y. Yang, and T. Li, "Finite-time synchronization of memristor-based neural networks with mixed delays," Neurocomputing, vol. 235, pp. 83-89, 2017. 